\title{
MORE ABOUT THE COMPARISON OF LOCAL AND NON-LOCAL NN INTERACTION MODELS
}

\author{
A. Amghar ${ }^{a}$, B. Desplanques ${ }^{\text {b }}$ \\ ${ }^{a}$ Faculté des Sciences, Université de Boumerdes, 35000 Boumerdes, Algeria \\ ${ }^{\mathrm{b}}$ Institut des Sciences Nucléaires (UMR CNRS/IN2P3-UJF), \\ F-38026 Grenoble Cedex, France
}

\begin{abstract}
The effect of non-locality in the NN interaction with an off-energy shell character has been studied in the past in relation with the possibility that some models could be approximately phase-shifts equivalent. This work is extended to a non-locality implying terms that involve an anticommutator with the operator $p^{2}$. It includes both scalar and tensor components. The most recent "high accuracy" models are considered in the analysis. After studying the deuteron wave functions, electromagnetic properties of various models are compared with the idea that these ones differ by their non-locality but are equivalent up to a unitary transformation. It is found that the extra non-local tensor interaction considered in this work tends to re-enforce the role of the term considered in previous works, allowing one to explain almost completely the difference in the deuteron D-state probabilities evidenced by the comparison of the Bonn-QB and Paris models for instance. Conclusions for the effect of the non-local scalar interaction are not so clear. In many cases, it was found that these terms could explain part of the differences that the comparison of predictions for various models evidences but cases where they could not were also found. Some of these last ones have been analyzed in order to pointing out the origin of the failure.
\end{abstract}

Key words: NN interaction, models, non-locality, deuteron form factors PACS: 13.40.Gp, 13.75.Cs, 21.30.Fe

Email address: desplanq@isn.in2p3.fr (B. Desplanques). 


\section{Introduction}

A major aim of electron scattering experiment on few-body systems is to validate the ingredients entering the description of the process. These include the NN interaction models and the associated currents. With this respect, the recent measurements of the deuteron form factors at JLab $[1,2]$ represent a valuable information. The interpretation of these data is not an easy one however. There are uncertainties on the nucleon form factors (see Ref. [3] for a recent review). The effect of relativistic corrections, which begin to show up in the explored $Q^{2}$ range, is approach dependent for a part which is not negligeable [4]. Some genuine effects, due to two-pion exchange, to an underlying quark structure of the constituents, to the field-theory foundation of the interaction ..., are expected to show up but they are likely to be mixed with other ones. Moreover, within a given scheme (corresponding to some equation for instance), NN interaction models, seemingly different, may represent the same physics. This involves different non-localities as expected from phaseshift equivalent potentials obtained by unitary transformations.

As is well known, within a non-relativistic approach, it is always possible to describe the NN interaction below the meson-production threshold by a local potential for each partial wave separately. However, theoretically, the interaction is expected to be non-local. This observation motivated a recent study on the role of the non-locality, essentially on a phenomenological basis [5]. The non-locality in interaction models has many sources and often results from accounting for a theoretical ingredient, explaining that it is not always emphasized by the authors. Part of it originates from relativistic corrections

( $\sqrt{m / e}$ factors, structure of Dirac spinors). It has been considered in Bonn models [6] and is sometimes approximated by linearly $p^{2} / m^{2}$ dependent terms which makes them tractable in configuration space (Paris [7], Bonn-R [6] and Nijmegen [8] models). Another part comes from the coupling of the dominant NN channel to other ones, $\Delta \Delta$ or $\pi N N$ components. In these cases, the original time non-locality is transformed into a spatial one. This non-locality is not explicitly present in the models (except of course for the energy-dependent ones, full-Bonn for instance) but may be accounted for in the fitted parameters. Finally, the compositeness of the nucleon or the exchanged meson can also produce some non-locality. It is explicit in quark based calculations (see Ref. [9] for a recent work together with earlier references) but, most often, as in the previous case, it is hidden in the parametrization of the potential model.

While different non-localities are present in various models, it is not clear whether it helps in describing the NN interaction. These local and non-local models may be as many different models, in which case discriminating between them is important. One cannot exclude however that they represent 
the same physics for some part. In this case, there is not so much to study at the NN level and, most probably, one should look at other observables. In the example of form factors, a preferred model would require less corrections from two-body currents beside the genuine ones. The possibility to have different models representing the same physics was discussed in the past by Friar $[10,11$. For a long time, it was just a curiosity till it was realized that more accurate models, namely Paris [7] and Bonn-QB [6], could realize to some extent this conjecture $[12,13]$. Terms under consideration have an off-shell character and mainly concern the tensor component of the interaction. When these models were used with the corresponding currents, it turned out that the discrepancies between the predictions for electromagnetic observables tend to decrease. Some was left however at the same time that some difference in the deuteron D-state probability was unexplained. It was mentioned in Refs. $[12,13]$ that some role could be played by terms which have the form of an anticommutator of the kinetic energy with a tensor operator, $p^{2} \tilde{W}_{T}+\tilde{W}_{T} p^{2}$. Interestingly, Von Geramb et al. [14], using inverse scattering methods, were able to derive local equivalent potentials for the two Paris and Bonn-QB models, evidencing very close deuteron D-state probabilities, suggesting that the above term could also be important in making the predictions of the two models closer to each other. These results largely motivate the present paper.

Besides non-local contributions of the type, $i\left(p^{2} U-U p^{2}\right)$, which have been studied in a previous paper and later on by de Forest [15], we therefore consider non-local contributions of the type, $p^{2} \tilde{W}+\tilde{W} p^{2}$. As in the other case, $W$ can have a scalar as well as a tensor component. The first one may originate from overall relativistic $\sqrt{m / e}$ normalization factors [16], mostly ignored in non-relativistic approaches. Due to the cancellation between the attractive and the repulsive components of the force due to $\sigma(2 \pi)$ and $\omega$ exchanges, its effect is not expected to be very large. A larger effect may be due to the different Lorentz structures of these couplings, implying that the $p^{2} / m^{2}$ corrections to each of them add together instead of cancelling. The effect, which in relativistic mean-field approaches tends to decrease the $\sigma$ exchange contribution has a net repulsion. It is part of many models $[7,6,8]$ and we believe that it is theoretically relevant. Its size is not well known however and, as far as we can see, there was no systematic attempt until now to look for an optimized strength. Perhaps, a reason for this is that such a term can always be transformed away by a unitary transformation as we recalled. If it has some relevance, one should expect that a smaller number of parameters be necessary to fit the same body of data. An indication in this sense is given by the comparison of the two Nijmegen models, Nij1 and Nij2 [8]. Approximately based on the same philosophy (vertex form factors, ...), but differing by a linearly $p^{2}$ dependent term, they reproduce equally well NN scattering data $\left(\chi^{2}\right.$ per datum $\left.=1.03\right)$. The first one, which is non-local, requires however less parameters (41 instead of 47). The non-local component with a tensor character is primarily due to the pion exchange. This is a well established one 
as far as the long-range force is concerned.

When studying the effect of non-local terms of the form, $p^{2} \tilde{W}+\tilde{W} p^{2}$, we will consider a transformation up to first order in the interaction. Contrary to off-shell effects generated by the terms, $i\left(p^{2} U-U p^{2}\right)$, the determination of the unitary transformation at the lowest order is not straightforward. It requires to solve an integral equation, which can only be performed numerically. When this is done, it is possible to calculate the effect on observables and see whether such terms can explain discrepancies between models evidencing different non-localities. This concerns the comparison between models. For our purpose, it is not necessary to look at the corrections to the interaction model which result from eliminating some non-locality. We assume that the fit of the model to scattering data has accounted for them, at least to a good approximation. We nevertheless notice that such corrections were explicitly considered in the past (see Ref. [17] for a recent work). Ultimately, one is interested in a comparison with experiment. With this last respect, the previous comparison may be useful to discriminate between effects that are simply due to the mathematical representation of the model and those due to genuine aspects such as a two-pion-exchange contribution [6,7], the choice of the strong $\pi N N$ coupling $[18,19], \ldots$

The plan of the paper will be as follows. In the second section, we present the non-local terms considered in this work and show how they can be transformed away by a unitary transformation at the lowest order. This involves two terms with a scalar and a tensor character. The effect on wave functions is discussed in the third section for the deuteron case, including both its $\mathrm{S}$ and $\mathrm{D}$ state components. The fourth section is devoted to the effect on the deuteron form factors and the structure functions, $A\left(Q^{2}\right)$ and $B\left(Q^{2}\right)$ as well as the tensor polarization, $T_{20}\left(Q^{2}\right)$. This part incorporates effects with an off-shell character described in an earlier work. Comparison of predictions for different interaction models is made when correcting them for a difference in their non-locality. A few cases where the discrepancy between predictions is not explained, and sometimes enhanced, are examined. A discussion and a conclusion are given in the fifth section.

\section{Formalism}

Non-locality can be produced by various contributions to the interaction. That one we are considering here originates from an expansion of the spinors entering the meson-exchange interaction. The part, which is by far the best identified, is due to the pion exchange. Its center of mass expression, up to first order terms in $\mathbf{p}^{2} / M^{2}$, is given by: 


$$
\begin{aligned}
& V^{\pi}\left(\mathbf{p}^{\prime}, \mathbf{p}\right)=-g_{\pi N N}^{2} K_{\pi N N}^{2}\left(\mathbf{k}^{2}\right) \frac{\overrightarrow{\tau_{1}} \cdot \overrightarrow{\tau_{2}}}{\mu_{\pi}^{2}+\mathbf{k}^{2}} \\
& \times\left(\frac{\overrightarrow{\sigma_{1}} \cdot \mathbf{k} \overrightarrow{\sigma_{2}} \cdot \mathbf{k}}{4 M^{2}}-\frac{\overrightarrow{\sigma_{1}} \cdot \mathbf{k} \overrightarrow{\sigma_{2}} \cdot \mathbf{k}}{16 M^{4}}\left(\mathbf{p}^{\prime 2}+\mathbf{p}^{2}\right)\right. \\
& \left.\quad-\frac{\overrightarrow{\sigma_{1}} \cdot\left(\mathbf{p}^{\prime}-\mathbf{p}\right) \overrightarrow{\sigma_{2}} \cdot\left(\mathbf{p}^{\prime}+\mathbf{p}\right)+\overrightarrow{\sigma_{2}} \cdot\left(\mathbf{p}^{\prime}-\mathbf{p}\right) \overrightarrow{\sigma_{1}} \cdot\left(\mathbf{p}^{\prime}+\mathbf{p}\right)}{32 M^{4}}\left(\mathbf{p}^{\prime 2}-\mathbf{p}^{2}\right)\right) .
\end{aligned}
$$

The above equation may be written as:

$$
V^{\pi}=V_{S}^{\pi}+V_{T}^{\pi}+\left\{\frac{\mathbf{p}^{2}}{M}, \tilde{W}_{S}^{\pi}\right\}+\left\{\frac{\mathbf{p}^{2}}{M}, \tilde{W}_{T}^{\pi}\right\}+\left[\frac{\mathbf{p}^{2}}{M}, i U^{\pi}\right]
$$

It can be extended to the exchange of other mesons:

$$
V=V_{S}+V_{T}+\left\{\frac{\mathbf{p}^{2}}{M}, \tilde{W}_{S}\right\}+\left\{\frac{\mathbf{p}^{2}}{M}, \tilde{W}_{T}\right\}+\left[\frac{\mathbf{p}^{2}}{M}, i U\right]
$$

In the above expression, $\tilde{W}_{S}$ and $\tilde{W}_{T}$ can be expanded in such a way to exhibit their spin-isospin structure. In configuration space, they would read:

$$
\begin{aligned}
& \tilde{W}_{S}=\sum_{s, t} W_{S}^{s, i}(r) P_{s, i}, \\
& \tilde{W}_{T}=\sum_{t} W_{T}^{i}(r) S_{12}(\hat{r}) P_{1, i},
\end{aligned}
$$

where the operators $P_{s, i}$ represent the projectors on different spin and isospin channels. The spin tensor operator, shortly written $S_{12}$, is defined as $S_{12}(\hat{r})=$ $\overrightarrow{\sigma_{1}} \cdot \hat{r} \overrightarrow{\sigma_{2}} \cdot \hat{r}-\overrightarrow{\sigma_{1}} \cdot \overrightarrow{\sigma_{2}} / 3$. Together with this definition, we write the deuteron wave function as $\left|\psi(r)>\propto\left[u(r)+3 w(r) / \sqrt{8} S_{12}(\hat{r})\right]\right|^{3} S_{1}>$. Working with states of given spin and isospin, these projectors can be omitted as well as the corresponding indices. The spin-tensor operator cannot be however. Without loss of generality, we can therefore make the replacements:

$$
\tilde{W}_{S} \leftrightarrow W_{S}\left(=W_{S}(r)\right), \quad \tilde{W}_{T} \leftrightarrow W_{T} S_{12}\left(=W_{T}(r) S_{12}(\hat{r})\right) .
$$

The two first terms in Eq. (3), $V_{S}$ and $V_{T}$, represent the standard scalar and tensor parts of the non-relativistic interaction. These terms are local and are present in all interaction models. They differ by the precise value of the coupling constant, $g_{\pi N N}$, and the expression of the hadronic form factor. This one is taken as 1 like in the Reid models or assumes a more realistic form like in most other models. The potentials that only retain the two first terms at the r.h.s. of Eqs. $(2,3)$ are considered as local ones. They in particular include the so-called RSC[20], Reid93, Nij2 [8] and Argonne V18 [21] models that will be evoked in this work. They are denoted here as type-I models. 
The so-called Paris [7], Nij1 and Nij93 [8] models contain a minimal nonlocality given by a term like the third one in Eq. (3). Partly justified by its appearance at the first order in $\mathbf{p}^{2} / M^{2}$, the limitation to this term is also motivated by the fact that it can be dealt with relatively easily in configuration space. These models, like the type-I ones, are generally built in configuration space. They are denoted as type-II models. Interestingly, these models tend to show a suppression of the deuteron S-wave at short distances smaller than local models corresponding to a similar description of the short range part of the interaction. An open question is to know whether this can be related to the non-local term proportional to $\tilde{W}_{S}$ in Eq. (3).

A last class of models (denoted type-III) is represented by the Bonn ones, QA, QB, QC, CD [6]. Contrary to the models discussed above, they consider the full structure of the Dirac spinors describing on mass-shell nucleons. Due to this, these models contain all kinds of non-locality present in Eq. (2) for the simplest pion-exchange case and in Eq. (3) for the most general case. They in particular involve terms that have an off-shell character and were studied in earlier works $[12,13,15]$. The dominant contribution was provided by the tensor part of $U^{\pi}$ which could explain 2/3 of the difference in the deuteron D-state propabilities evidenced by the comparison of calculations performed with the Paris and Bonn-QB models. An open question here, indirectly answered by von Geramb [14], is to know whether the fourth term in Eq. (3), $\propto \tilde{W}_{T}$, can explain the remaining part.

In comparing models, it is important that they are physically equivalent with respect to NN scattering properties. The so-called "high accuracy" models, Nij1, Nij2, Argonne V18 and Bonn-CD should therefore retain our attention. Concerning effects in relation with the tensor part of the interaction, we will also include the Paris and Bonn-QB models which were used in a previous work. These two models provide values for the mixing angle, $\epsilon_{1}$, which are relatively close to each other on the one hand, and close to the experimental ones on the other.

While the good quality of the fit to NN scattering data is essential to avoid biases in the analysis we are going to perform, the failure to establish some relationship between the various in principle phase-shift equivalent models can have different origin. Beyond higher order corrections, it may reveal a genuine physical effect. Although it can be largely accounted for in the model fitting, the two-pion exchange contribution explicitly incorporated in the Paris model could be an example of such an effect. It may also reveal that some ingredient in a model is, on the contrary, physically irrelevant. Moreover, but not independently, it may suggest that models are not completely determined by scattering data in the range where they are available. Looking for possible failures can thus provide information on the underlying physics, which, after all, is the ultimate goal for studying the NN interaction properties. Anticipating 
on the following, this aspect will mainly involve its scalar part. In the next subsections, we show how non-local terms present in Eq. (3) can be transformed away at the lowest order in $\mathbf{p}^{2} / M^{2}$. This is done successively for the scalar and tensor components of the interaction. We will concentrate on the terms that have an anticommutator character, $\left\{\mathbf{p}^{\mathbf{2}} / M, \tilde{W}_{S}\right\}$ and $\left\{\mathbf{p}^{\mathbf{2}} / M, \tilde{W}_{T}\right\}$ in Eq. (3). The other terms of the commutator type, $\left[\mathbf{p}^{\mathbf{2}} / M, i U\right]$, which are relatively easy to deal with, were discussed elsewhere $[12,13,15]$.

For definiteness, we denote by $H$ and $H^{\prime}$ the two Hamiltonians that, in the ideal case, would be related to each other by a unitary transformation:

$$
H^{\prime}=e^{S} H e^{S^{+}}
$$

where $S=-S^{+}$. The Hamiltonian, $H$, will be that one that contains a nonlocal term, $V_{N L}$ which we want to transform away by making a unitary transformation and $H^{\prime}$ will be the resulting Hamiltonian. The two Hamiltonians therefore assume the following expressions:

$$
\begin{aligned}
H & =\frac{p^{2}}{M}+V+V_{N L} . \\
H^{\prime} & =\frac{p^{2}}{M}+V^{\prime},
\end{aligned}
$$

When the non-local term, $V_{N L}$, is transformed away to make it local, the interaction $V$ acquires an extra contribution $\Delta V$, defined as:

$$
\Delta V=V^{\prime}-V
$$

Starting from a two-body interaction, $\Delta V$ will generally involve two-body but also many-body contributions. As to the solutions of the Hamiltonians, $H$ and $H^{\prime}$, they are denoted $\Psi$ and $\Psi^{\prime}$.

\subsection{Non-locality in the scalar part of the interaction models}

In this subsection, we consider two models that differ by a non-local term proportional to $W_{S}$. The non-local term, $V_{N L}$, therefore assumes the following expression:

$$
V_{N L}=\left\{\frac{\mathbf{p}^{2}}{M}, W_{S}(r)\right\}
$$

The physical origin of the last term in Eq. (10) has been briefly mentioned in the introduction. The first candidate for such a contribution is provided by the 
examination of the $\omega$ and $\sigma$ ( $2 \pi$ in a S-state) exchanges. The cancellation that occurs for these contributions at the zeroth order in $p / M$ is absent at the order $(p / M)^{2}$, hence a possible sizeable term. In comparison, the contribution due to the pion exchange in Eq. (2) contains extra factors of the order $(p / M)^{2}$. Due to the above cancellation, minimal relativity factors $\sqrt{m / e}$, whose effect was studied in a first step by Elster et al. [16], may only be a part of the dominant contribution. Non-locality could also arise from eliminating degrees of freedom involving mesons in flight or baryons excitations. It however appears that the corresponding spatial and time non-localities, individually large, tend to cancel each other. This probably explains the success of a local potential in reproducing the spectrum of the Bethe-Salpeter equation [22].

Equation (6) together with the expression of $V_{N L}$ given by Eq. (10) cannot be solved easily but taking into account that the term we want to remove is of order $(p / M)^{2}$, we can solve it order by order by making an expansion of the unitary transformation that we denote $e^{S_{S}}$. At the lowest order in the interaction, we have thus to fulfill the following equation:

$$
\left\{\frac{\mathbf{p}^{2}}{M}, W_{S}\right\}+\left[S_{S}, \frac{\mathbf{p}^{2}}{M}\right]=\Delta V_{S}^{0}
$$

where $\Delta V_{S}^{0}$ must be local. This is achieved by choosing $S_{S}$ as:

$$
S_{S}=i\left(\frac{\vec{p} \cdot \vec{r} V_{0}(r)+V_{0}(r) \vec{r} \cdot \vec{p}}{2}\right)
$$

where the function $V_{0}$ satisfies the following first-order differential equation:

$$
V_{0}(r)+\vec{r} \cdot \vec{\nabla} V_{0}(r)=W_{S}(r)
$$

The solution of this equation is given by the following integral:

$$
V_{0}(r)=-\frac{1}{r} \int_{r}^{\infty} W_{S}\left(r^{\prime}\right) d r^{\prime}
$$

The integral can be calculated numerically provided that the integrand is not too singular. The singular character of $V_{0}$ due to the presence of the front factor $1 / r$ is partly removed by the presence of the factor, $\vec{r}$, in the expression of $S_{S}$, Eq. (12). It is likely that this singular character is the consequence of limiting ourselves to the lowest order terms in both expanding $e^{S_{S}}$ and in the interaction. It should be removed in a complete calculation but this supposes a fully non-local unitary transformation. 
While the non-local term in Eq. (3) is removed at the lowest order, an extra local contribution to the interaction with same order in the coupling constant is generated. Denoted $\Delta V_{S}^{0}$ in Eq. (11), its expression is given in the appendix. Let's precise here that its locality holds for each partial wave separately, as expected from phase-shift equivalent models. On the other hand, it is somewhat more singular than the interaction models we started from or contains terms with extra powers of $1 / r$. By fitting the model parameters to the scattering NN data, such terms may be partly accounted for but we do not expect to completely explain the discrepancy between different models, the new terms having a functional form that is generally discarded in building models. Finally, an important difference is to be noticed with the treatment of the non-local term of the commutator type, $\left[\mathbf{p}^{\mathbf{2}} / M, i U\right]$, made elsewhere $[12,13]$. The correction, $\Delta V$, contains in the present case a contribution of the first order in the coupling $g^{2}, \Delta V^{0}$, while it was of the second order for this other term.

For practical applications, we will consider the two potential models, Nij1 and Nij2, which have been built with approximately the same ingredients, except evidently for the presence of a non-local term in the first one, which has the form of the term considered in this work. We also considered other couples of models, Paris and RSC for instance. Their probing strength as to their possible unitary equivalence was not found very strong. Instead, the comparison pointed out to a discrepancy having its source in the different values for the quantities, $A_{S}$ and $a_{t}$ (and possibly the ratio $\eta=A_{D} / A_{S}$ ) obtained with these

models. Some correlation between $A_{S}$ and $a_{t}$ is well known but a correlation to $A_{D} / A_{S}$, which appears to be supported by the examination of various models, is not so. Characterizing the asymptotic behavior, the difference evidenced by the comparison of these values that are observable ones, cannot be removed by the unitary transformation considered here.

\subsection{Non-locality in the tensor part of the interaction models.}

In this subsection, we consider two models that differ by a non-local tensor term of the anticommutator type:

$$
V_{N L}=\left\{\frac{\mathbf{p}^{\mathbf{2}}}{M}, W_{T}(r) S_{12}(\hat{r})\right\}
$$

The non-local tensor term of the commutator type has been discussed at length in earlier works $[12,13]$.

To eliminate the non-local term under consideration, we proceed as in the previous subsection, with the same approximations (expansion of the uni- 
tary transformation, now denoted $e^{S_{T}}$, up to first order in $S_{T}$ and first-order terms in the interaction retained). The quantity $S_{T}$ has to be chosen in such a way that its commutator with the kinetic energy term $\mathbf{p}^{2} / M$ cancels the term $\left\{\mathbf{p}^{2} / M, W_{T} S_{12}\right\}$ without generating the same terms as those we want to remove. This implies to solve an equation similar to Eq. (11) for the scalar case:

$$
\left\{\frac{\mathbf{p}^{2}}{M}, W_{T} S_{12}\right\}+\left[S_{T}, \frac{\mathbf{p}^{2}}{M}\right]=\Delta V_{T}^{0}
$$

where $\Delta V_{T}^{0}$ has to be local. This supposes to take two terms in $S_{T}$ and is achieved by:

$$
\begin{aligned}
S_{T}=\frac{i}{2}(\vec{p} \cdot \vec{r} & S_{12}(\hat{r}) V_{1}(r) \\
& \left.\quad+\left(\overrightarrow{\sigma_{1}} \cdot \vec{p} \overrightarrow{\sigma_{2}} \cdot \vec{r}+\overrightarrow{\sigma_{2}} \cdot \vec{p} \overrightarrow{\sigma_{1}} \cdot \vec{r}-\frac{2}{3} \overrightarrow{\sigma_{1}} \cdot \overrightarrow{\sigma_{2}} \vec{p} \cdot \vec{r}\right) V_{2}(r)+h . c .\right),
\end{aligned}
$$

The functions, $V_{1}(r)$ and $V_{2}(r)$, have to satisfy the following relations:

$$
\begin{aligned}
r\left(V_{1}^{\prime}(r)+V_{2}^{\prime}(r)\right) & =W_{T}(r), \\
V_{1}(r)+V_{2}(r)+\left(r V_{2}(r)\right)^{\prime} & =0 .
\end{aligned}
$$

Despite it is more complicated than Eq. (13), the above system of equations can be formally solved with the result:

$$
\begin{aligned}
& V_{2}(r)=-\frac{1}{r} \int_{r}^{\infty} d r^{\prime} \int_{r^{\prime}}^{\infty} \frac{W_{T}\left(r^{\prime \prime}\right)}{r^{\prime \prime}} d r^{\prime \prime}, \\
& V_{1}(r)=-V_{2}(r)-\frac{1}{r} \int_{r}^{\infty} \frac{W_{T}\left(r^{\prime}\right)}{r^{\prime}} d r^{\prime} .
\end{aligned}
$$

Numerical values for $V_{1}(r)$ and $V_{2}(r)$ are then obtained by performing successively the integrations in Eqs. (20) and (21). Concerning the singularities entering these expressions, the same observations as for $V_{0}(r)$ can be made here.

Similarly to the scalar case, the removal of the non-local tensor term of the anticommutator type generates an extra contribution to the interaction, $\Delta V_{T}^{0}$, with a local character. Its expression is given in the appendix. Again, it evidences an analytic structure different from what is usually included in potential models. A large part of it is certainly accounted for by fitting its parame- 
ters to scattering data. Whether the remaining part can show up is probably difficult to answer in view of other uncertainties.

Applications will be made for the couple of the Paris and Bonn-QB models that we considered in a previous work for part of the non-local tensor component with a commutator type, assuming implicitly that non-local scalar terms of the anticommutator type are more or less the same. We also considered the couple of models Argonne V18 and Bonn-CD. In this case, a non-local scalar term is also included, slightly obscuring the analysis.

\section{Comparison of wave functions}

In this section, we compare wave functions obtained from various models differing by their non-locality. In principle, and as far as the models are phase-shift equivalent, it should be possible to relate the corresponding wave functions according to the equality:

$$
\Psi^{\prime}=e^{S} \Psi
$$

where $\Psi$ and $\Psi^{\prime}$ are solutions of Schrödinger equations with Hamiltonians respectively given by Eqs. (7) and (8).

Consistently with our approximation retaining the first term in the expansion of the unitary transformation $e^{S}$ made in its determination, we assume that the correction to $\Psi$ produced by the application of this transformation is given by

$$
\Delta \Psi=S \Psi
$$

In practice, we will calculate this quantity, add it to $\Psi$ and compare the result to the wave function obtained independently in a different model, $\Psi^{\prime}$, which it could be compared with. Expressions of $\Delta \Psi$ in the case of a non-local term with an off-shell character, $\propto i U$, have been given in Refs. [12,13]. We here give these expressions for the other non-local terms, $\propto W_{S}$ and $\propto W_{T}$.

Correction to the wave function from transforming away the non-local term $\left\{\mathbf{p}^{2} / M, W_{S}\right\}$

For the first case, one gets separately for the $S$ and $D$ wave components of the deuteron wave function:

$$
\Delta u=\frac{1}{2} W_{S} u+V_{0} r u^{\prime}
$$




$$
\Delta w=\frac{1}{2} W_{S} w+V_{0} r w^{\prime}
$$

Using the expression of $V_{0}$ in term of $W_{S}$, Eq. (13), one can easily check that the correction to the norm, always at the first order in $S$, is zero separately for the $S$ and $D$ waves:

$$
\int_{0}^{\infty} d r u \Delta u=\int_{0}^{\infty} d r w \Delta w=0 .
$$

This result is consistent with the unitary character of the transformation and the fact it does not mix the two waves.

Correction to the wave function from transforming away the non-local term $\left\{\mathbf{p}^{2} / M, W_{T} S_{12}\right\}$

Expressions for the case of the term $\propto W_{T}$ are more complicated:

$$
\begin{aligned}
\Delta u= & \frac{\sqrt{8}}{3}\left(\left(V_{1}+5 V_{2}+\frac{1}{2} W_{T}\right) w+\left(V_{1}+2 V_{2}\right)\left(r w^{\prime}-w\right)\right), \\
\Delta w= & \frac{\sqrt{8}}{3}\left(\left(V_{1}-V_{2}+\frac{1}{2} W_{T}\right) u+\left(V_{1}+2 V_{2}\right)\left(r u^{\prime}-u\right)\right) \\
& -\frac{2}{3}\left(\left(V_{1}+2 V_{2}+\frac{1}{2} W_{T}\right) w+\left(V_{1}+2 V_{2}\right)\left(r w^{\prime}-w\right)\right) .
\end{aligned}
$$

As previously, using the above expressions, one can check that the correction to the norm is zero but the result now holds for the total sum of the $S$ and $D$ waves contributions:

$$
\int_{0}^{\infty} d r(u \Delta u+w \Delta w)=0 .
$$

Comparison of wave functions for Nij1 and Nij2 models

In Figs. 1 and 2, we show the effect of the transformation discussed above in the case of a scalar non-local term, $\left\{\mathbf{p}^{2} / M, W_{S}\right\}$. This is done for the couple of models Nij1 and Nij2 that precisely differ by such a term. As far as the unitary transformation is truncated to the first order term in $S$, applying the transformation $e^{S}$ on $\Psi$ or the inverse transformation $e^{-S}$ on $\Psi^{\prime}$ is not equivalent. We therefore present results where the two cases are considered in the same figure. Beyond $0.8 \mathrm{fm}$, where corrections are small enough to be treated perturbatively, it is found that both the $\mathrm{S}$ and $\mathrm{D}$ waves become closer to the wave functions they should be compared with (see the insert). Some discrepancy remains at the largest distances. In the D-wave case, it partly reflects a difference in the asymptotic normalizations, $A_{D}$, that are not affected by the unitary transformation. In the S-wave case, it is not clear whether the 


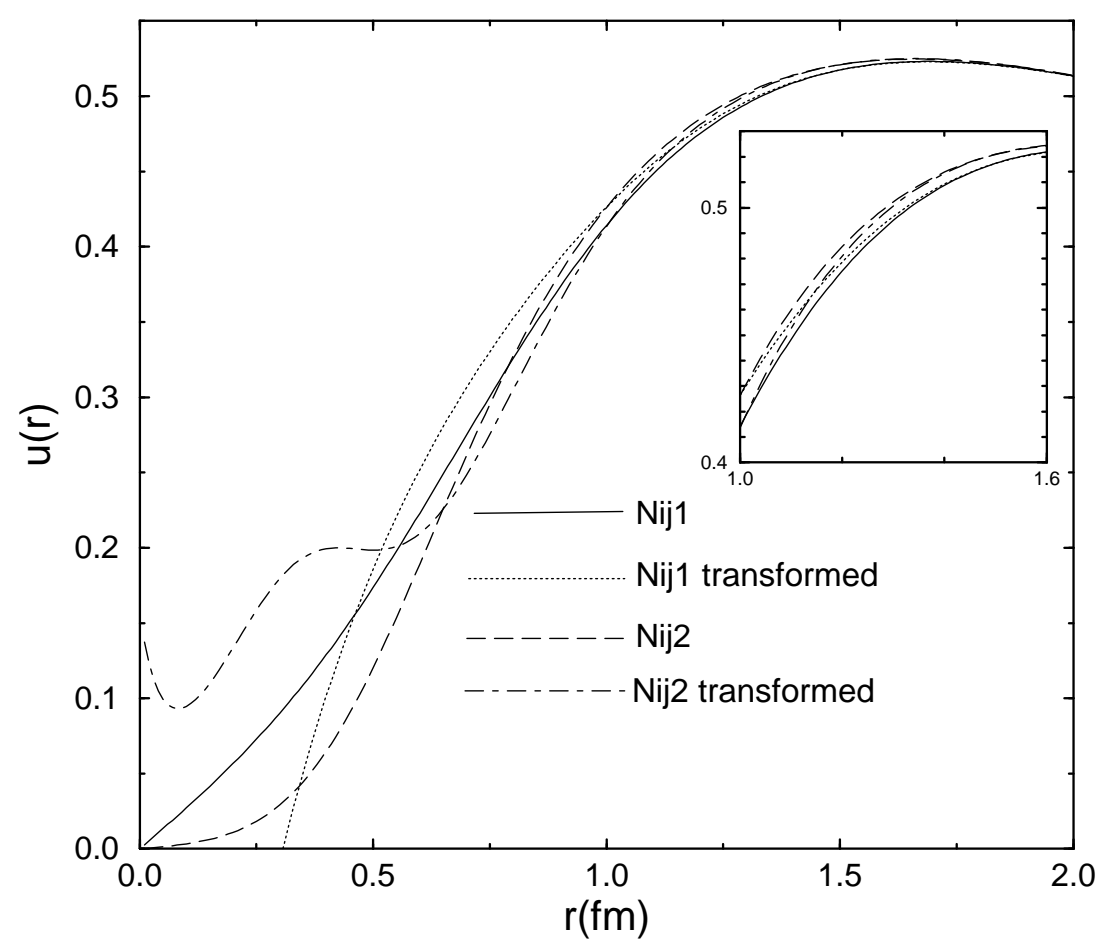

Fig. 1. Effect of a non-local scalar term, $\left\{\mathbf{p}^{2} / M, W_{S}\right\}$, on the deuteron S-waves: The effect is shown for the Nij1 and Nij2 models. The corresponding deuteron S-waves, $u(r)$, are represented by the continuous and dashed lines respectively. They should be compared with the transformed waves obtained from the other model, represented in the figure by dashed-dotted and dotted lines. The insert shows an enlarged part of the figure in the region where the non-locality effect shows up at large distances. For this calculation, $W_{S}$ is taken from the Paris model.

remaining difference around $1.4 \mathrm{fm}$ is an indirect consequence of the previous one, of the choice of $W_{S}$ (actually taken from the Paris model in a first step analysis) or simply of the model parametrization. It is noticed that the wave functions at these distances are likely to be sensitive to the medium range of the interaction, which itself involves scattering data at intermediate energies. The effect of a difference in reproducing these data, such as the $8 \%$ one for the $\epsilon_{1}$ mixing angle around $100 \mathrm{MeV}$ for the two models considered here, cannot be removed, evidently, by the unitary transformation.

At lower distances, examination of the figures, especially for the S-wave, evidences structures. They are due for a large part to the perturbative character of our approach. At the lowest distances, the effect of the transformation goes in the right direction. It therefore seems reasonable to attribute the smoother behavior of the S-wave for some models to their $p^{2}$ dependence. The effect is however too large. It is likely that a more accurate treatment would transfer part of the effect at larger distance, around $0.6 \mathrm{fm}$, where the present effect is too small or even has a sign opposite to what was expected. This transfer is not arbitrary however as the norm should remain unchanged. 


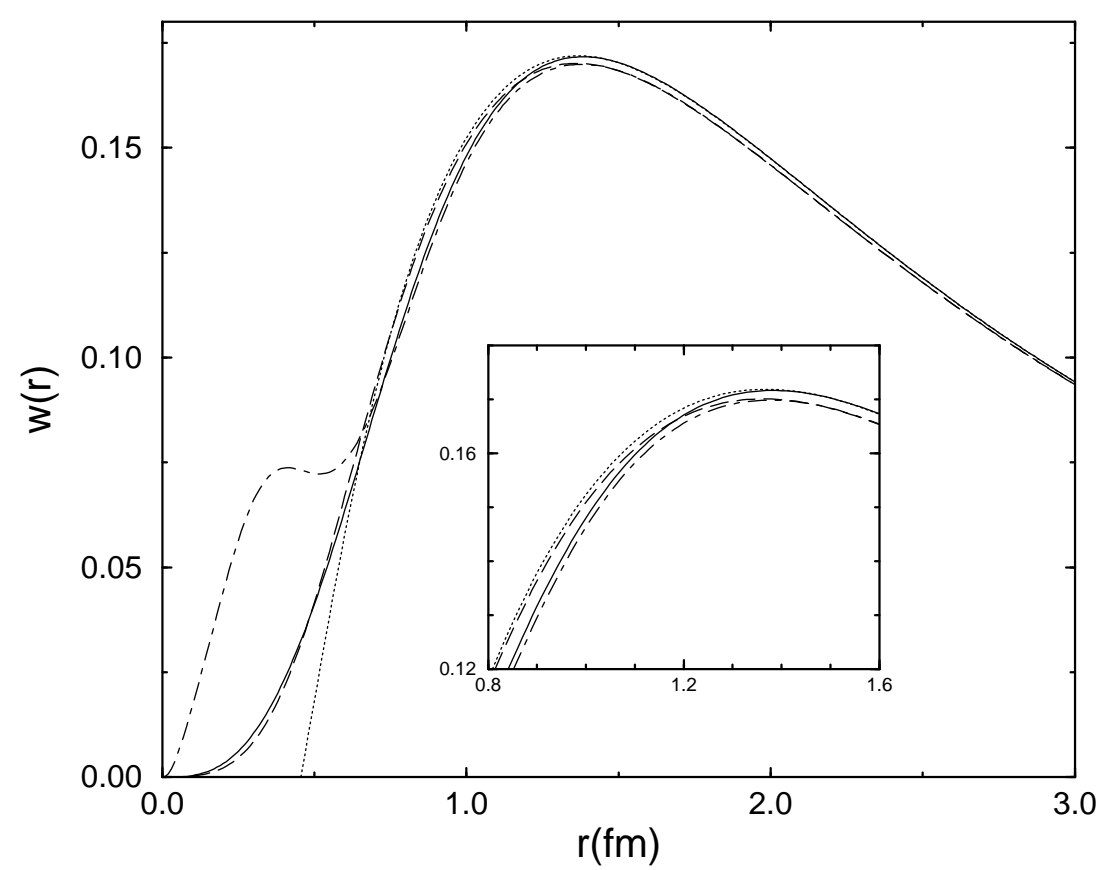

Fig. 2. Effect of a non-local scalar term, $\left\{\mathbf{p}^{2} / M, W_{S}\right\}$, on the deuteron D-waves: The effect is shown for the Nij1 and Nij2 models. Same as in Fig. 1, but for the deuteron D-waves, $w(r)$.
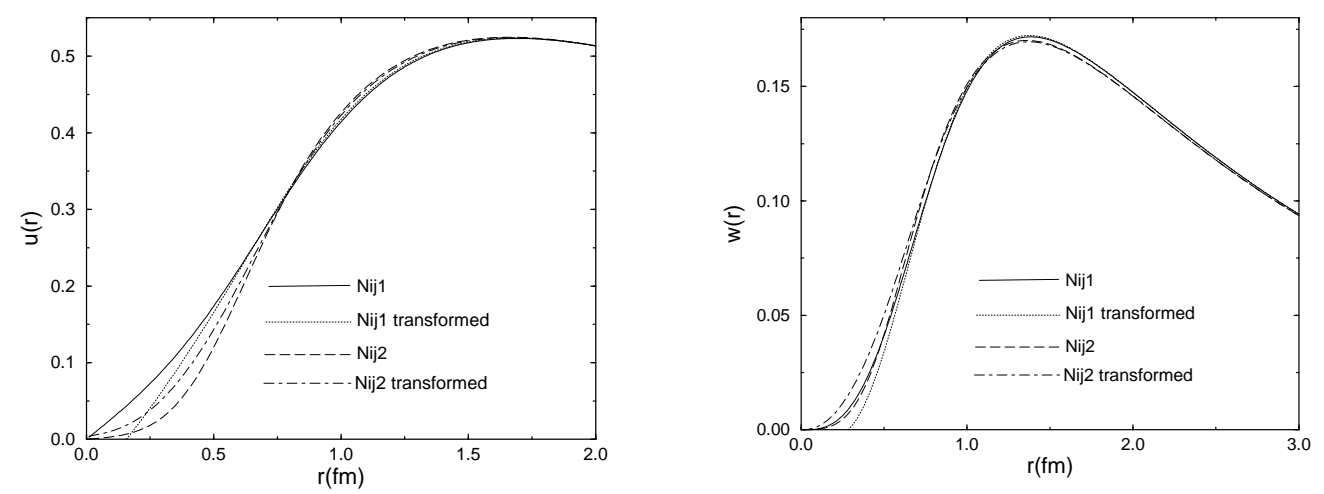

Fig. 3. Effect of a non-local scalar term, $\left\{\mathbf{p}^{2} / M, W_{S}\right\}$, on the deuteron S- and D-waves: The effect is shown for a different $W_{S}$ in calculating the transformed S- and D- waves from the Nij1 and Nij2 models. The corresponding curves are respectively given in the right and left parts; same as in Fig. 1 otherwise. For this calculation, $W_{S}$ is taken from a meson-exchange model.

The existence of the above structures may introduce a bias in the comparison of form factors and other observables that will be made in the next section. Actually, the effect is limited by the fact that in calculating the form factors, we only retain the linear corrections in order to preserve the norm, according to Eqs. (26) and (29). Moreover, in the integrand, this correction is multiplied by a wave function which tends to zero in the relevant domain. The actual effect on form factors would thus involve a factor $q^{2} r^{3} / 24$ in the integrand and is consequently suppressed. It is roughly of the order $q^{2} r_{0}^{4} / 96$ where $r_{0}$ 


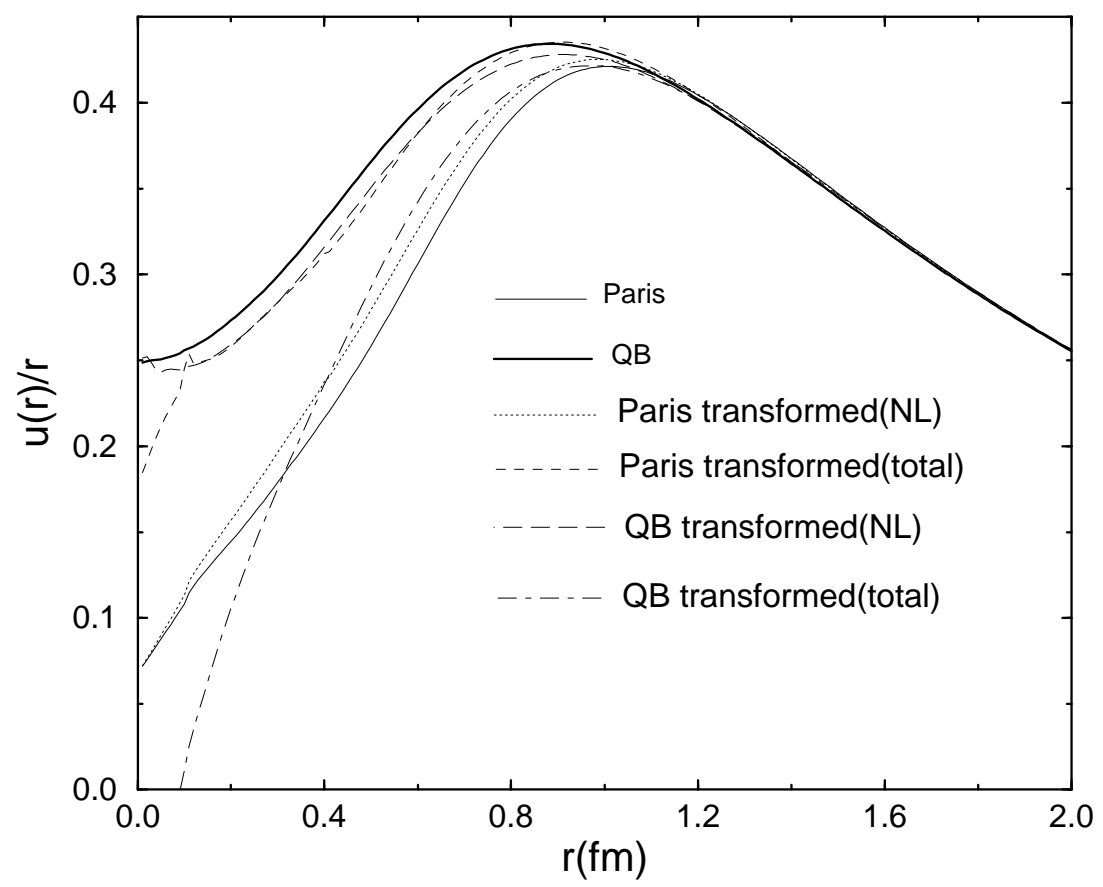

Fig. 4. Effect of a non-local tensor term together with off-shell contributions: The effect is considered for the S-waves of the Paris and Bonn-QB models. Curves show the quantity $u(r) / r$ to better emphasize the short range behavior. The thin and thick continuous lines represent the corresponding quantity for these models. The transformed wave functions accounting for the non-local tensor term, $\left\{\mathbf{p}^{2} / M, W_{T} S_{12}\right\}$, are given respectively by dotted and long-dashed lines (labelled NL). The wave functions accounting for the whole effect are given by the short-dashed and dash-dotted lines. These last ones should be compared with the wave function of the other model.

is of the order of $0.4 \mathrm{fm}$. Furthermore, one cannot exclude cancellations with corrections at higher $r$. As will be seen in the next section, it is what occurs and the different structures should be considered consistently.

Due to the theoretical uncertainty on $W_{S}$, we estimated the transformed wave functions with assuming that this quantity was produced by single meson exchanges $(\pi, \rho, \omega, \sigma)$ with standard values for the parameters. Results are presented in Fig. 3. They evidence smaller structures than in Figs. 1 and 2, probably in relation with a smaller strength of the $W_{S}$ term. By using in the following a term taken from the Paris model, it is likely that its strength is not underestimated.

Comparison of wave functions for Bonn- $Q B$ and Paris models

In Figs. 4 and 5, we show the effect of the transformation discussed above in the case of a tensor non-local term, $\left\{\mathbf{p}^{\mathbf{2}} / M, W_{T} S_{12}\right\}$. For this calculation, $W_{T} S_{12}$ is taken as the sum of a $\pi$-exchange contribution appearing in Eq. (1), which provides the dominant contribution, and a $\rho$-exchange contribution, which has a cut-off role. Together with this non-local term, we consider the effect of the off-shell term studied in a previous work. A monopole cut-off 


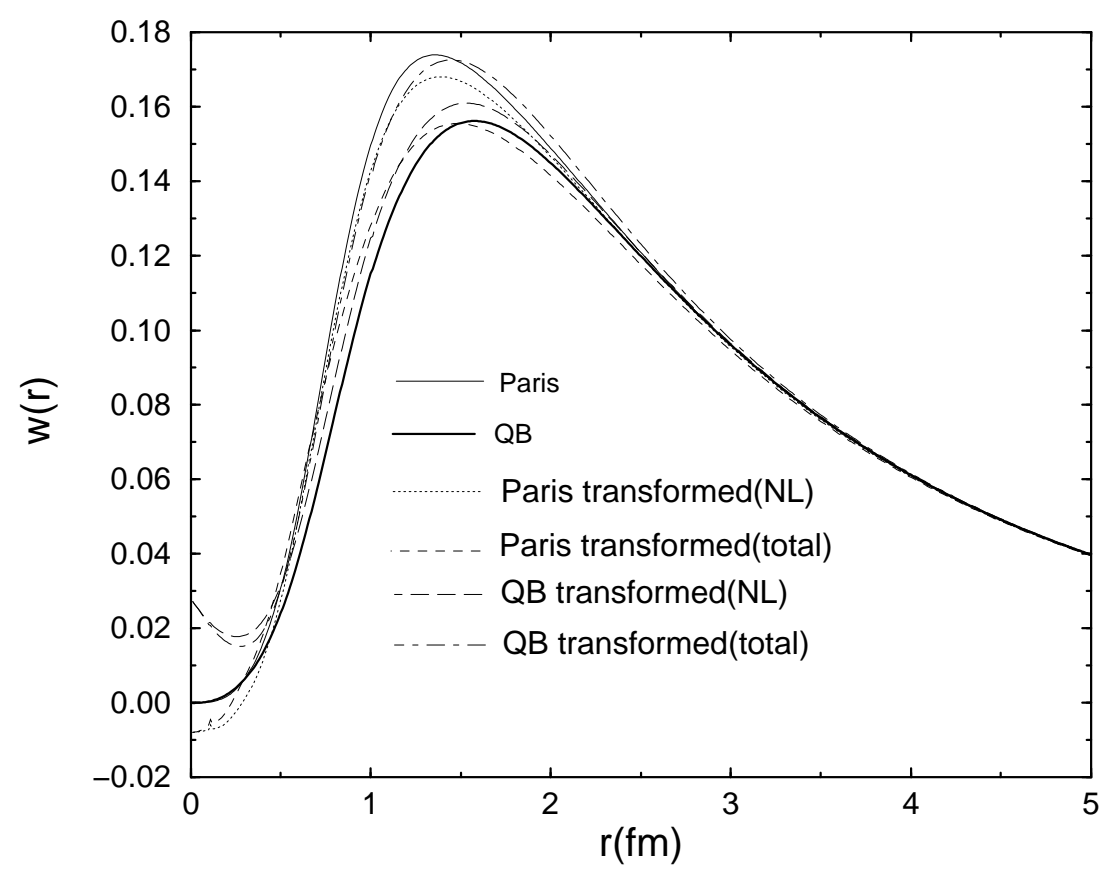

Fig. 5. Effect of a non-local tensor term together with off-shell contributions: The effect is considered for the D-waves of the Paris and Bonn-QB models. Curves represent the usual D-waves, $w(r)$; same as in Fig. 4 otherwise.

form factor, $\left(\Lambda^{2}-\mu^{2}\right) /\left(\Lambda^{2}+k^{2}\right)$, with $\Lambda=1.05 \mathrm{GeV}$, is introduced in all calculations [13] so that to kill the short-range part of the interaction, for which the analysis developped in this work is more uncertain, both theoretically and mathematically. For the S-wave (Fig. 4), the transformed wave functions are relatively close to the one they should be compared with. The effect of the first term, $\left\{\mathbf{p}^{\mathbf{2}} / M, W_{T} S_{12}\right\}$, which arises from the action on the D-wave (therefore different of the scalar case) goes in the right direction. Most of the effect comes from the term with an off-shell character. Notice that for this curve, we plotted $u(r) / r$, in order to better see the effects at short distances. Quite similar results hold for the D-wave shown in Fig. 5. It is immediate from these results that when considering the electromagnetic properties of the deuteron, the predictions of the different models will tend to be close to each other when the currents that are associated to each model are accounted for. Concerning the little structures appearing in the transformed wave functions, the remarks made previously for the comparison of Nij1 and Nij2 wave functions could be repeated here. The amplitude of these structures is much less important here however. 


\section{Effect on deuteron form factors and electromagnetic observables}

Given a physical system and an underlying theory, it can be described by an infinity of interaction models, depending on the choice of degrees of freedom. This is quite similar to the choice of a frame or a basis in making an experiment or a calculation. This freedom is at the origin of the non-observable character

of the wave function. Concerning the system itself, this is realized by using phase-shift equivalent potentials. As for the interaction with an external probe, described by an operator denoted $O$, the equivalence of the models implies a relation such as:

$$
\langle\Psi|O| \Psi\rangle=\left\langle\Psi^{\prime}\left|O^{\prime}\right| \Psi^{\prime}\right\rangle .
$$

In this expression, the operator $O^{\prime}$, which can contain in the minimal case onebody terms but also many-body ones, will here refer to the electromagnetic interaction. Using the expression relating $\Psi^{\prime}$ and $\Psi$, Eq. (22), it is found that the above equality supposes that:

$$
\begin{aligned}
O^{\prime} & =e^{S} O e^{-S}=O+\Delta O, \\
\Delta O & =[S, O]+\ldots
\end{aligned}
$$

In the case where $O$ is a one-body operator, it is immediately found that the correction at the r.h.s. has in the minimal case a two-body character. In order to make a relevant comparison of predictions of observables from different representations, one has therefore to account for the contribution due to $\Delta O$ when calculations are performed. This is what we are doing in this section. The question of making a comparison to measurements, which in any case supposes to consider the contribution of other currents, is evoked in the conclusion.

For the off-shell correction considered in Refs. [12,13], the commutator $\Delta O$ could be calculated explicitly, leading to two-body currents that, except for a factor, had for some part a structure identical to the pair-term contribution to the charge operator. The expression for the magnetic operator could not be related to any known two-body operator. A similar situation holds for the transformation considered here for terms of the form $\left\{\mathbf{p}^{2} / M, \tilde{W}_{S, T}\right\}$. One thus gets the following contribution for the isoscalar charge operator in configuration space at the lowest order :

$$
\begin{aligned}
{[S, O]=\frac{i}{2} e^{i \vec{q} \cdot \vec{r} / 2} } & \left(\vec{q} \cdot \vec{r} V_{0}(r)+\vec{q} \cdot \vec{r} S_{12}(\hat{r}) V_{1}(r)\right. \\
& \left.+\left(\overrightarrow{\sigma_{1}} \cdot \vec{q} \overrightarrow{\sigma_{2}} \cdot \vec{r}+\overrightarrow{\sigma_{2}} \cdot \vec{q} \overrightarrow{\sigma_{1}} \cdot \vec{r}-\frac{2}{3} \overrightarrow{\sigma_{1}} \cdot \overrightarrow{\sigma_{2}} \vec{q} \cdot \vec{r}\right) V_{2}(r)\right) .
\end{aligned}
$$


In practice, calculations can be performed indistinctively in two ways. The first one, in the spirit of equivalent models, consists in calculating the matrix element of $O+\Delta O$ with the wave function, $\Psi^{\prime}$, and compare it to the matrix element calculated with $O$ together with the wave function, $\Psi$. In the second one, the first matrix element is replaced by the matrix element of $O$ with the transformed wave function, $e^{-S} \Psi^{\prime}$. While in this later approach conclusions immediately stem from the examination of transformed w.f. discussed in the previous section, the former approach is not without interest. Indeed, some of the corrections to charge, quadrupole and magnetic form factors (for the scalar part $W_{S}$ ) have a relatively simple expression:

$$
\begin{aligned}
\Delta F_{C}\left(Q^{2}\right)= & G_{E}^{S}\left(Q^{2}\right) \int_{0}^{\infty} d r x j_{1}(x)\left(\left(u^{2}(r)+w^{2}(r)\right) V_{0}(r)\right. \\
& \left.+\frac{2 \sqrt{8}}{3}\left(u(r) w(r)-\frac{1}{\sqrt{8}} w^{2}(r)\right)\left(V_{1}(r)+2 V_{2}(r)\right)\right) \\
\Delta F_{Q}\left(Q^{2}\right)= & \frac{6 \sqrt{2}}{Q^{2}} G_{E}^{S}\left(Q^{2}\right) \int_{0}^{\infty} d r \\
\times & \left(\left(3 j_{2}(x)-x j_{1}(x)\right)\left(u(r) w(r)-\frac{1}{\sqrt{8}} w^{2}(r)\right) V_{0}(r)\right. \\
+ & \sqrt{8} j_{2}(x)\left(-u^{2}(r)+w^{2}(r)+\frac{1}{\sqrt{2}} u(r) w(r)\right) V_{2}(r) \\
+ & \frac{\sqrt{2}}{3}\left(3 j_{2}(x)-x j_{1}(x)\right) \\
\times & \left.\left(u^{2}(r)+\frac{3}{2} w^{2}(r)-\sqrt{2} u(r) w(r)\right)\left(V_{1}(r)+2 V_{2}(r)\right)\right) \\
\Delta F_{M}\left(Q^{2}\right)= & \frac{3}{4} G_{E}^{S}\left(Q^{2}\right) \int_{0}^{\infty} d r 3 j_{2}(x) w^{2}(r) V_{0}(r) \\
+ & \left(3 j _ { M } ^ { S } ( Q ^ { 2 } ) \int _ { 0 } ^ { \infty } d r \left(x j_{1}(x)\left(u^{2}(r)-\frac{1}{2} w^{2}(r)\right)\right.\right. \\
& \left.\left.x j_{1}(x)\right)\left(w^{2}(r)+\sqrt{2} u(r) w(r)\right)\right) V_{0}(r)+\ldots
\end{aligned}
$$

where $x=Q r / 2$. The above expressions can be also obtained from those using the transformed wave functions by performing an integral by parts and taking into account that the term $\ldots . .\left.\right|_{0} ^{\infty}$ is zero. Contrary to these ones however, the integrand is quite smooth and does not evidence structures at short distances. Far to suggest numerical inaccuracies or errors in the formalism, the structures that we discussed in the previous section have to be taken as they are and, most important, dealt with consistently. The dots at the last line of the expression for the magnetic form factor contain terms with the derivatives of the wave 
functions. Unlike the charge and quadrupole form factors, these ones cannot be removed using an integration by parts.

When comparing observables, we will proceed in two ways, depending on which model predictions are compared with. This is dictated by the first order approximation made in expanding the unitary operator $e^{S}$, which is required for consistency, as explained above, but prevents to keep the unitary character of the transformation. In assessing the two sets of results, it is likely that the truth should be in between. We successively consider the static observables, the form factors and finish the section by mentioning a few peculiar results.

\subsection{Static observables}

Effect of $\left\{\mathbf{p}^{2} / M, W_{S}\right\}$

When comparing predictions for models differing by a term $\left\{\mathbf{p}^{\mathbf{2}} / M, W_{S}\right\}$, we found very small effects for the static observables, $r_{D}^{2}$ and $Q_{D}$, and obviously none for $\mu_{D}$ (in relation in this case with the absence of change of $P_{D}$ ). The non-local term being short-range-behaved, we rather expect effects in the dynamical observables (form factors) as it can be inferred from the discussion of the wave functions in Sect. 3. Concerning the models, Nij1 and Nij2, we looked at more specifically, static observables are quite close to each other, leaving little room for possible effects. The difference in the values of $r_{D}^{2}$, $0.004 \mathrm{fm}^{2}$, can be traced back to a difference in the asymptotic normalizations $A_{S}$ for $75 \%$ (models roughly fulfill the relation $\Delta r_{m} \simeq 1.9 \Delta A_{S}$ ). What we calculated, $\simeq 0.001 \mathrm{fm}^{2}$, is consistent with the unexplained part. For the quadrupole moment, we found a correction that has a sign opposite to what would be required by the comparison of the two models. Again, the major part of the difference in this case is likely to have another origin (see our discussion of the D wave around $1.4 \mathrm{fm}$ in Fig. 2, Sect. 3). As for the the magnetic moment, it is unchanged since the normalizations of the S- and D-waves are separately unchanged.

Effect of $\left\{\mathbf{p}^{2} / M, W_{T} S_{12}\right\}$ and other terms

Effects of the tensor term, $\left\{\mathbf{p}^{\mathbf{2}} / M, W_{T} S_{12}\right\}$, are significantly more important than for the scalar one. In a previous work [13], we could explain 2/3 of the difference in the D-state probabilities for the Bonn-QB and Paris models $(0.78 \%)$ by considering a non-local tensor term with an off-shell character, $\left[\mathbf{p}^{\mathbf{2}} / M, i U\right]$. By adding the above term, we can now explain a difference of $0.74 \%$ ( $0.14 \%$ for the new term alone). The deuteron D-wave probability enters in the magnetic moment according to the relation $\Delta \mu_{D}=3 / 2\left(\mu_{S}-1 / 2\right) \Delta P_{D}$. The difference for the two models is $\Delta \mu_{D}$ is $0.0045 \mathrm{~nm}$ while we calculate $0.0043 \mathrm{~nm}$. Another observable sensitive to the tensor force is the quadrupole moment. Considering the quantity $Q_{D} /\left(A_{S} A_{D}\right)$, that may be less sensitive 
to differences in $A_{S}$ or $A_{D}$ left unchanged by the unitary transformation we are dealing with, we found a difference of $0.19 \mathrm{fm}^{3}$ or $0.20 \mathrm{fm}^{3}$ depending on which model is corrected. The difference in the predictions is $0.23 \mathrm{fm}^{3}$. Finally, we explain $60 \%$ of the difference in the squared charge radius evidenced by the comparison of the two models, Bonn-QB and Paris. This last observable is directly related to the slope of the deuteron structure function, $A\left(Q^{2}\right)$.

As far as static observables are concerned, it thus appears that they are very close to each other once the effects of their supposed unitary equivalence are taken into account.

\subsection{Comparison of form factors with models Nij1 and Nij2, effect of the non- local scalar term, $\left\{\mathbf{p}^{2} / M, W_{S}\right\}$}

The effect of the non-local scalar term, $\left\{\mathbf{p}^{2} / M, W_{S}\right\}$, on electromagnetic observables is shown in three figures for the case of the Nij1 and Nij2 potentials models, which differ by such a term. Here and below, we expect that if this term has some relevance in explaining the difference in the wave functions of these models, then predictions for the observables should be closer to each other when the appropriate currents are used. The ratio of these predictions, that we represent in the figures, should therefore approach 1.
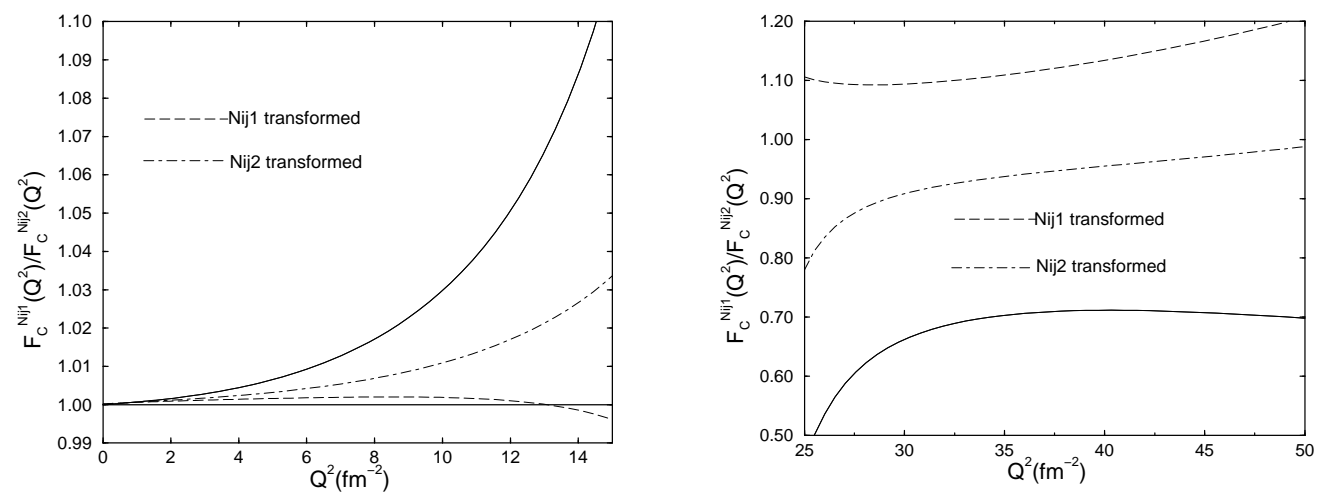

Fig. 6. Effect of a non-local term, $\left\{\mathbf{p}^{2} / M, W_{S}\right\}$, on charge form factors: Curves represent the ratio of the Nij1 to the Nij2 charge form factors from $Q^{2}=0$ to $Q^{2}=15 \mathrm{fm}^{-2}$ for the left part and from $Q^{2}=25$ to $Q^{2}=50 \mathrm{fm}^{-2}$ for the right part. The ratio of the uncorrected predictions is given by the continuous line. The ratios with the transformed Nij1 and Nij2 w.f. (or including the associated two-body currents) are respectively given by the dashed and the dash-dotted lines.

The effect on charge form factors is seen in Fig. 6 that has been split into two parts, due to the appearance of a zero in the range $15-25 \mathrm{fm}^{-2}$. While the effect was hardly significant for static observables, examination of the figure indicates that it is quite relevant at higher $Q^{2}$ and goes in the right direction. 

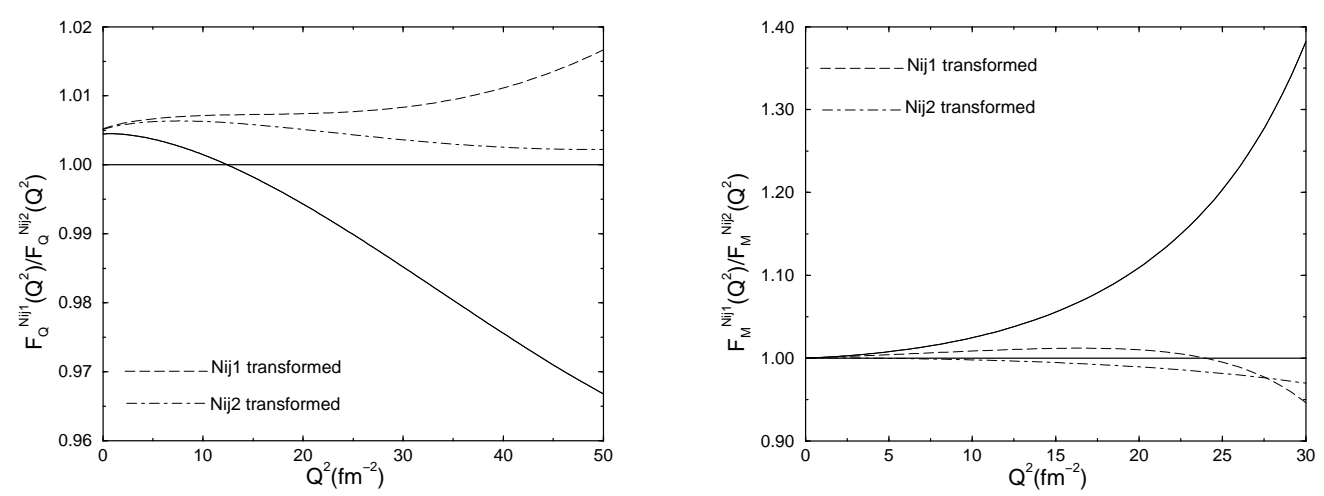

Fig. 7. Effect of a non-local term, $\left\{\mathbf{p}^{2} / M, W_{S}\right\}$, on quadrupole and magnetic form factors: Curves represent the ratio of the Nij1 to the Nij2 quadrupole and magnetic form factor from $Q^{2}=0$ to $Q^{2}=50 \mathrm{fm}^{-2}$ for the first one (left part) and $Q^{2}=0$ to $Q^{2}=30 \mathrm{fm}^{-2}$ for the second one (right part). They are defined as in Fig. 6 .
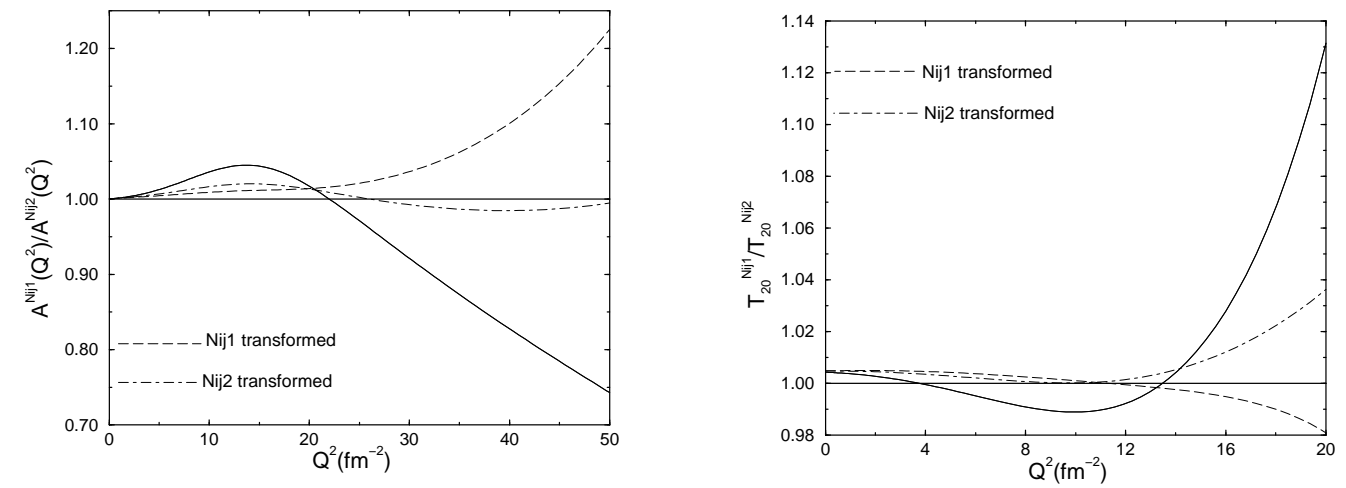

Fig. 8. Effect of a non-local term, $\left\{\mathbf{p}^{2} / M, W_{S}\right\}$, on $A\left(Q^{2}\right)$ and $T_{20}\left(Q^{2}\right)$ observables: Curves represent the ratio of the Nij1 to the Nij2 $A\left(Q^{2}\right)$ and $T_{20}\left(Q^{2}\right)$ observables, respectively for the left and right parts. They are defined as in Fig. 6.

Results do not depend much on whether two-body currents are added to one model or to the other. A similar conclusion holds for the quadrupole form factor as well as the magnetic one shown in Fig. 7. Notice that the last one is given up to $30 \mathrm{fm}^{-2}$ due to the presence of a zero at a slightly larger $Q^{2}$.

It is also instructive to look at quantities which are closer to what is measured, namely the deuteron structure function $A\left(Q^{2}\right)$, and the tensor polarization at $\theta_{e}=70^{0}, T_{20}\left(Q^{2}\right)$. They are given in Fig. 8. Not surprisingly, they nicely confirm the above conclusions.

Results quite similar to the above ones have been obtained when extended to other interaction models (Nij1 replaced by Paris or Nij93 ; Nij2 replaced by Argonne V18 or Reid93). The agreement is especially good for those models that better fit the NN scattering data. Due to the various uncertainties already mentioned, we will mainly retain that the currents associated with the removal of a term, $\left\{\mathbf{p}^{2} / M, W_{S}\right\}$, in the interaction can reasonably account for differences in model predictions, both in sign and in magnitude. Some differ- 
ences remain however (and hopefully) unexplained. They will be discussed at the end of this section.

\subsection{Comparison of form factors with models Bonn-QB and Paris, effect of} the non-local tensor term
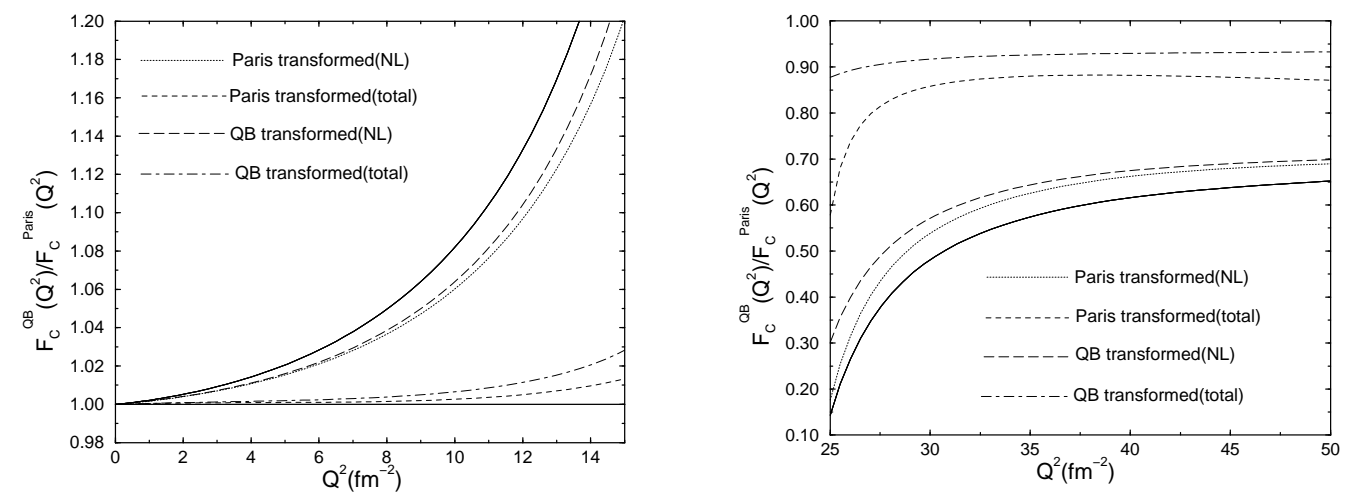

Fig. 9. Effect of a non-local term, $\left\{\mathbf{p}^{2} / M, W_{T} S_{12}\right\}$ together with off-shell effects for the charge form factor: Curves represent the ratio of the Bonn-QB to the Paris charge form factors from $Q^{2}=0$ to $Q^{2}=15 \mathrm{fm}^{-2}$ for the left part and from $Q^{2}=25$ to $Q^{2}=50 \mathrm{fm}^{-2}$ for the right part. The ratio of the uncorrected predictions is given by the continuous line. The ratio when the Paris (Bonn-QB) w.f. is transformed for the first ( $\left\{\mathbf{p}^{\mathbf{2}} / M, W_{T} S_{12}\right\}$ ) and second (off-shell) non-locality is given by the dotted and short-dashed (long-dashed and dash-dotted) lines.
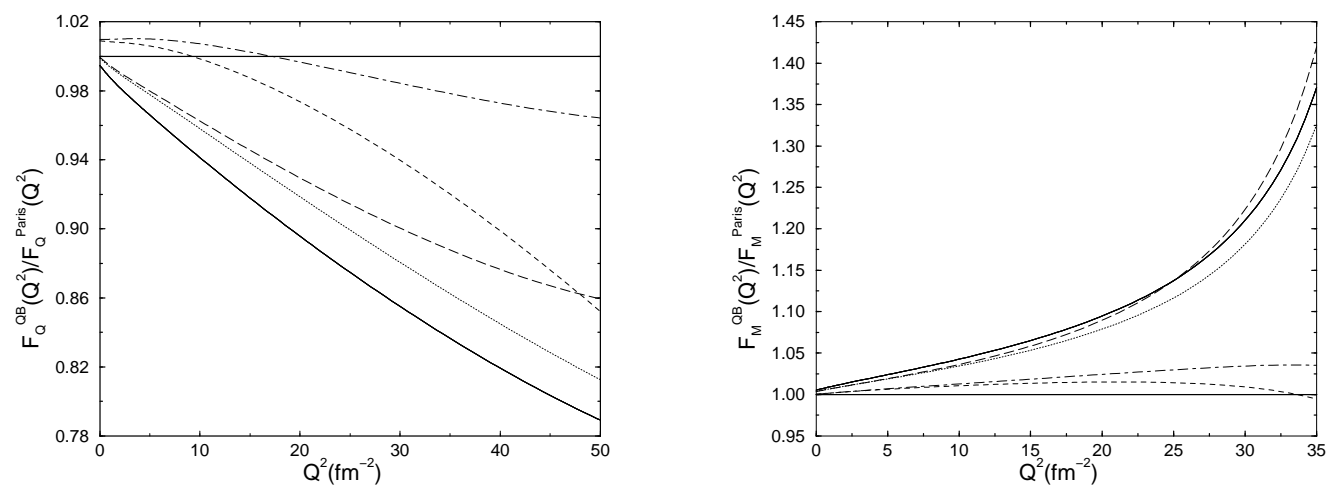

Fig. 10. Effect of a non-local term, $\left\{\mathbf{p}^{2} / M, W_{T} S_{12}\right\}$ together with off-shell effects for the quadrupole and magnetic form factors: Curves represent the ratio of the Bonn-QB to the Paris quadrupole and magnetic form factors (left and right parts respectively). They are defined as in Fig. 9.

The effect of a tensor term like $\left\{\mathbf{p}^{2} / M, W_{T} S_{12}\right\}$ on electromagnetic observables is shown in three figures for the case of the Bonn-QB and Paris models, which differ by such a term. These models also differ by terms that have an off-shell character and were studied in an earlier work. In presenting the various effects, we first present the new one considered here and then add the older one. 

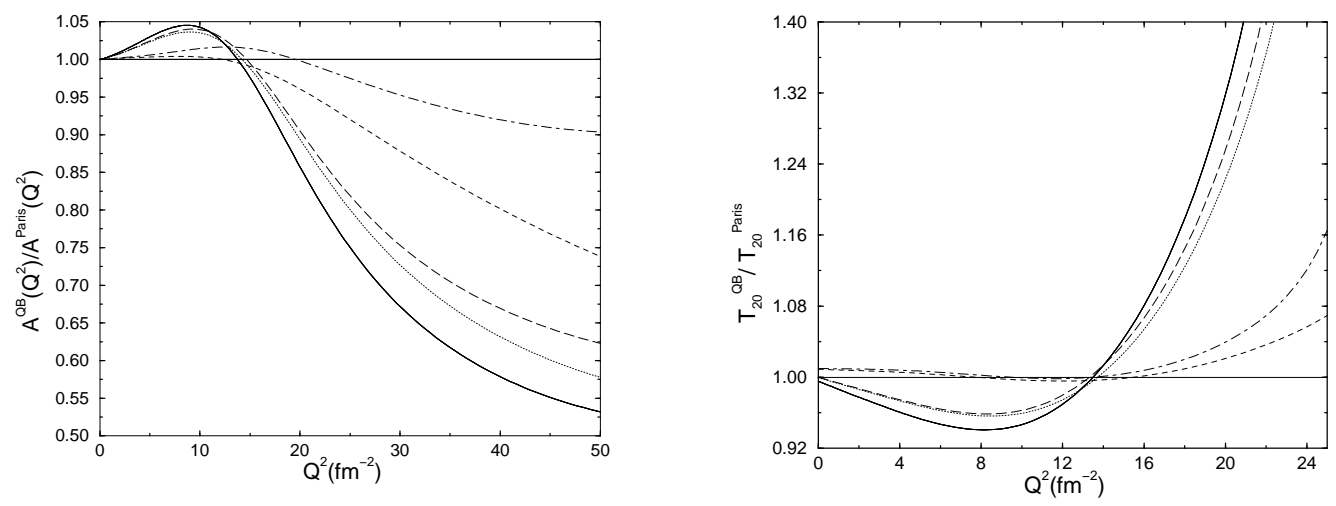

Fig. 11. Effect of a non-local term, $\left\{\mathbf{p}^{2} / M, W_{T} S_{12}\right\}$ together with off-shell effects for $A\left(Q^{2}\right)$ and $T_{20}\left(Q^{2}\right)$ observables: Curves represent the ratio of the Bonn-QB to the Paris $A\left(Q^{2}\right)$ and $T_{20}\left(Q^{2}\right)$ observables. They are defined as in Fig. 9.
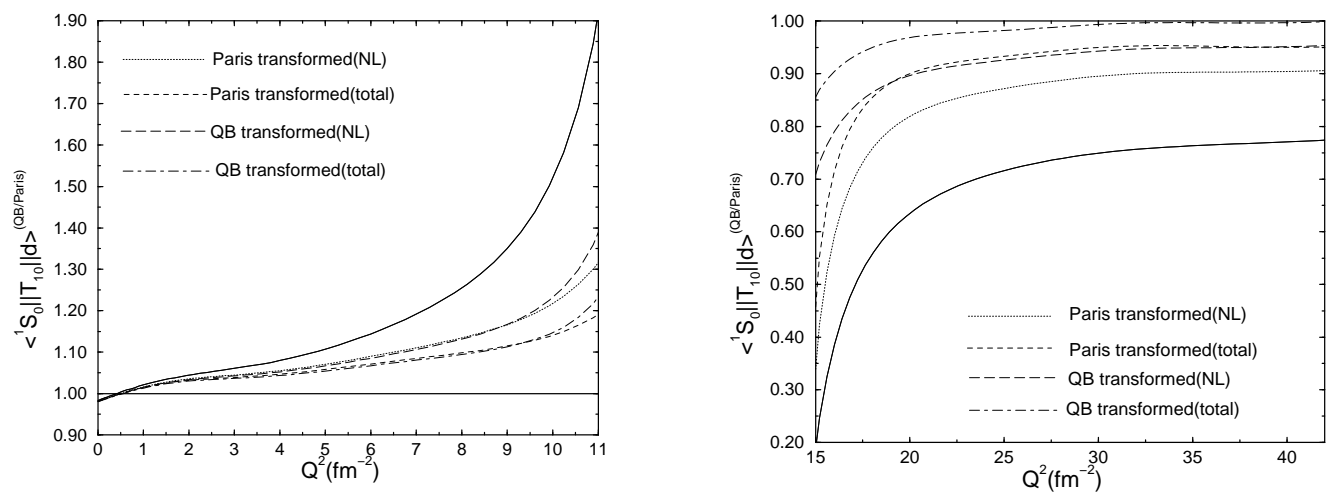

Fig. 12. Effect of a non-local term, $\left\{\mathbf{p}^{2} / M, W_{T} S_{12}\right\}$ together with off-shell effects for the deuteron electrodisintegration form factor near threshold: Curves represent the ratio of the Bonn-QB to the Paris form factor from $Q^{2}=0$ to $Q^{2}=11 \mathrm{fm}^{-2}$ for the left part and from $Q^{2}=15$ to $Q^{2}=40 \mathrm{fm}^{-2}$ for the right part. The ratio of the uncorrected predictions is given by the continuous line. The ratio when the Paris (Bonn-QB) w.f. is transformed for the first $\left(\left\{\mathbf{p}^{2} / M, W_{T} S_{12}\right\}\right)$ and second (off-shell) non-locality is given by the dotted and short-dashed (long-dashed and dash-dotted) lines.

The effect of the non-local tensor term on the charge form factor is presented in Fig. 9. It explains a little part of the difference between the Bonn-QB and Paris model predictions. By far, the dominant effect is due to the off-shell effect associated to the $\pi$ - and $\rho$-exchange tensor force. Quite similar results hold for the quadrupole and magnetic form factors shown in Fig. 10 and, as a consequence, for the structure function, $A\left(Q^{2}\right)$, and the tensor polarization, $T_{20}\left(Q^{2}\right)$, both given in Fig. 11. In these two cases, possible discrepancy is completely removed below $15 \mathrm{fm}^{-2}$. There may be still some beyond in relation with that one observed for the quadrupole form factor in Fig. 9. We notice that in dealing with the non-local tensor force, we omitted in the Bonn-QB model a term of the form:

$$
\sigma_{1} \cdot \mathbf{p}^{\prime} \times \mathbf{p} \quad \sigma_{2} \cdot \mathbf{p}^{\prime} \times \mathbf{p}
$$


This one appears at the fourth order in $p / M$ and, moreover, arising from vector-meson exchange, has a short-range behavior. In configuration space, this term gives rise to a $\overrightarrow{\sigma_{1}} \cdot \vec{L} \quad \overrightarrow{\sigma_{2}} \cdot \vec{L}$ term, which is present in the Paris model considered in this work, non-local terms that are similar to those we discussed and another non-local one, proportional to $\overrightarrow{\sigma_{1}} \cdot \vec{p} \overrightarrow{\sigma_{2}} \cdot \vec{p}[8]$. This last one could be dealt with by using a transformation identical to that given in Eq. (17), but with different relations of $V_{1}$ and $V_{2}$ to the potential strength (see appendix). At first sight, this new term could contribute in the right direction. However, in discussing its contribution, we should also include higher order contributions due to eliminating non-local terms at the lowest order, $\left[S, V_{N L}\right]$. These induced terms have an extra factor $1 / M^{2}$ (for the tensor part) but a range quite similar to that of vector-meson exchange.

Most results presented above concern an elastic transition. Some have been obtained for an inelastic transition, namely the deuteron electro-disintegration near threshold which involves a matrix element between the deuteron ${ }^{3} S_{1}\left(+{ }^{3} D_{1}\right)$ state and the ${ }^{1} S_{0}$ scattering state. They are presented in Fig. 12. Examination of these results shows features quite similar to the ones observed in other cases. In principle, this process allows one to get insight on the effect of a non-local term in a channel, ${ }^{1} S_{0}$, different from the deuteron one. However, as most of the effect is related to the tensor force, the conclusion one can draw from this case is limited.

As for the case of the non-local scalar term considered in the previous subsection, we looked at other models (Bonn-QB replaced by Bonn-CD ; Paris replaced by Nij1 or Nij93). Again, a better agreement is obtained with models that better fit NN scattering data. In the detail, a few points deserve to be discussed however.

\subsection{A few peculiarities}

The studies presented in this work were originally performed with the idea that various NN interaction models could be equivalent (up to a unitary transformation). With this respect, it is interesting that we can explain a large part of the differences between models. The question now arises to determine the limitations, if any. By construction, the models are not identical. Besides a difference in their non-locality, discussed throughout this work, there may be differences in the choice of various ingredients: coupling constants, vertex form factors, exchanged mesons together with their mass, quality of the fit to NN scattering data, number of free parameters, etc....After the non-locality effects have been removed, the comparison of model predictions can thus reveal more clearly features in relation with these ingredients. We here consider some of them. They involve a few percent effects that may not be significant at first 
sight but become so when appearing repeatedly. They also mainly concern the low momentum transfers, the high momentum transfer range being too sensitive to a variety of ingredients to make firm statements.
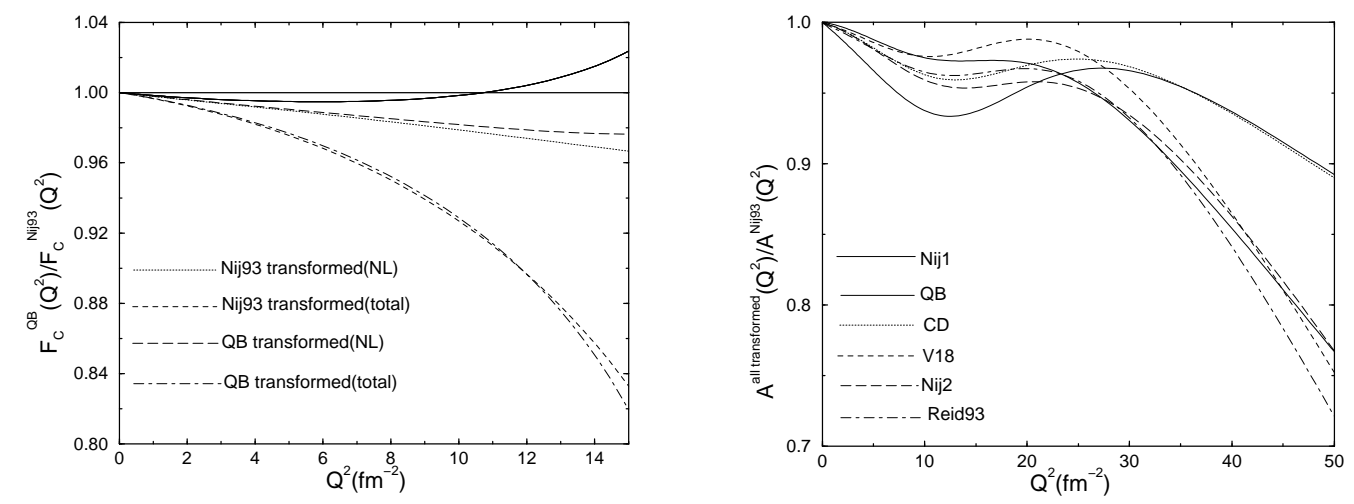

Fig. 13. Comparison to results involving the Nijmegen 93 interaction model, for the charge form factor (left part) and the $A\left(Q^{2}\right)$ structure function (right part).

In Fig. 13 (left part), we show an example where an approximate agreement between two models turns into a discrepancy when the influence of the nonlocal interaction is accounted for. It concerns the charge form factor calculated with the Bonn-QB and Nij93 models in the range $Q^{2}=0$ to $Q^{2}=15 \mathrm{fm}^{-2}$. Being alone, this example, which is given to illustrate our purpose, does not allow one to draw conclusions on one of the two models under consideration. However, after incorporating the non-locality effect, it appears that the charge form factor for the Nij93 model evidences the same pattern as for the other models. This can be seen in the comparison of the $A\left(Q^{2}\right)$ structure function calculated with the Nij93 model and with the set of models Nij1, Nij2, Reid93, Argonne V18 which, as already mentioned, provide very close results when non-locality effects are taken into account. The ratio of these quantities, shown in Fig. 13 (right part), evidences a difference in the slope or even a bump in the range $Q^{2}=0$ to $Q^{2}=15 \mathrm{fm}^{-2}$. This tends to suggest that the Nij93 model involves specific features that are absent in the other models. It is tempting to make a correlation with the fact that this model predicts a smaller radius than the other ones, whose effect could not be seen clearly without removing effects of the non-locality. As to the origin of this smaller radius, it could be due to the poorer quality of the fit to scattering NN data (only 15 parameters). Not independently, it could also be due to the presence in this model of a pomeron exchange contribution which is ignored in all the other models. Its long range is likely to produce specific features that could not be easily accounted for with shorter range contributions to the potential.

Another particular feature shows up when comparing the $A\left(Q^{2}\right)$ structure function calculated with the Paris model to that one calculated with one of models Nij1, Nij2, Reid93, Argonne V18 and Bonn-CD and the appropriate corrections for non-locality effects. The ratio, which is shown in Fig. 14 (right part), evidences a slight bump in the range $Q^{2}=0$ to $Q^{2}=15 \mathrm{fm}^{-2}$. A 

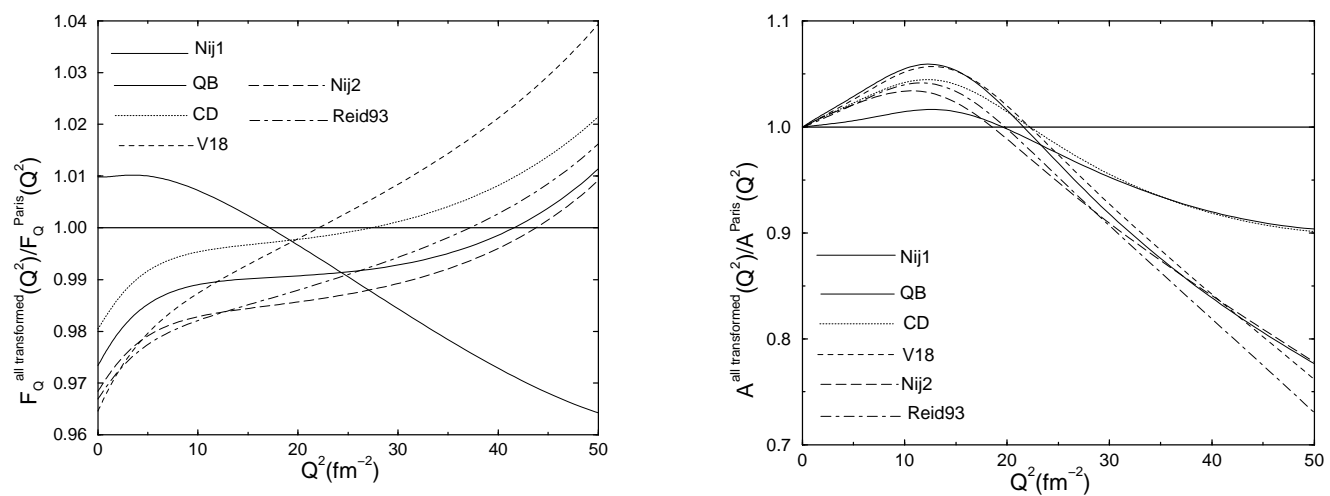

Fig. 14. Comparison to results involving the Paris interaction model, for the quadrupole form factor (left part) and the $A\left(Q^{2}\right)$ structure function (right part).

similar but less pronounced effect is seen with the Bonn-QB model. Again, the difference in the slope suggests to look at the deuteron radius predicted by the models. It is thus found that the radius for the Paris and Bonn-QB models, after accounting for non-locality effects, are slightly larger than for the other models. Before wondering about a possible origin for this difference in the radius, we emphasize another feature that may have some relation. It concerns the quadrupole form factor and more specifically the quadrupole moment. In many cases, we could explain differences in the quadrupole moment by the difference in the non-locality of the models. This is not the case for the Paris model (and to a lesser extent for the Bonn-QB model) as it can be seen in Fig. 14 (left part). The quadrupole moment for the Paris model is always larger than for the other models by an amount of $3-4 \%$. There is no direct relation to the radius discussed above although a larger value will certainly contribute to produce a larger quadrupole moment. An explanation could be looked for in the value of the $\pi N N$ coupling constant which is larger for the Paris (and Bonn-QB) model than for the other models which, for some of them, have been built on the prejudice of a value around $g_{\pi N N}^{2} / 4 \pi \simeq 13.5$ [18]. The value for the Paris model is closer to that one advocated by Ericson et al., $g_{\pi N N}^{2} / 4 \pi \simeq 14.11$ [19]. A larger coupling implies a larger attraction at large distances and therefore a larger probability to find the neutron and the proton far apart in the deuteron. In the case of the Paris model, one can add that the explicit two-pion exchange contribution is likely to also produce a larger attraction at long distances in comparison with an approximation where this contribution is treated as an exchange of an effective $\sigma$ with mass around $500 \mathrm{MeV}$.

\section{Conclusion}

In this work, we have studied the effect of non-local terms on the description of the NN interaction. We especially looked at terms which involve the anti- 
commutator with the operator $p^{2}$, namely $\left\{\mathbf{p}^{2} / M, \tilde{W}_{S, T}\right\}$. The present work completes another one where terms of the commutator form $\left[\mathbf{p}^{2} / M, i U\right]$, with an off-shell character, were considered. In dealing with these terms, we relied on an explicit form for the transformation allowing to get rid of them, which furthermore was treated at the first order in the interaction. In comparing models, this approach is probably not as good as an approach based on inverse scattering methods. It nevertheless has the advantage to be based on the explicit identification of non-local terms in interaction models and to provide an insight on the currents that have to be associated with the models. As far as we can see, the method is accurate enough for our purpose, especially in view of its qualitative character for a part. On the other hand, the study has been extended to other interaction models, allowing one to draw conclusions that the comparison of two models only could not.

For our study of the newly considered terms, we took the scalar part, $\tilde{W}_{S}$, from the Paris model while the tensor part, $\tilde{W}_{T}$, was taken from the sum of a contribution due to pion exchange, rather well determined, and a short range contribution. It is reminded that there is no a priori guarantee that these nonlocal terms can explain differences in interaction models which could have other sources and in some cases could not be explained (when they involve differences in observables such the asymptotic normalizations, $A_{S}$ and $A_{D}$ ).

We found that the scalar part, $\tilde{W}_{S}$, has quite small contributions to static quantities, such as the charge radius or the quadrupole moment. To evidence them, one has to get rid of contributions due to different asymptotic normalizations for instance, which compete with the effects we are looking at or are even larger. Some sizeable effect however shows up for dynamical observables such as form factors. It is difficult to make a definitive statement on the size of the effect in view of various uncertainties, but its sign and its magnitude can reasonably explain differences between models differing by the above term.

Concerning the tensor part, $\tilde{W}_{T}$, the contribution to static properties is more important. It, in particular, explains a non-negligeable part of the difference in the deuteron D-state probabilities between the Bonn models, which account for the full structure of Dirac spinors, and the other ones. When added to the effect of non-local terms with an off-shell character, which is larger, not much difference remains to be explained. For dynamical observables, the effect of the above term goes also in the right direction and accounts for a half of what could not be previously explained with the off-shell term. It would be interesting to see what would do an extra non-local tensor term omitted in our analysis. This one has a short range behavior but also a different structure which would require further theoretical elaboration.

While a large part of differences evidenced by the comparison of models with various non-localities can be ascribed to this feature, we would not like to 
give the impression that every difference can be explained that way. Interestingly, after removing the above effects, some differences between models, that were masked until now, more clearly appear. The correlation between the slope of the deuteron structure function, $A\left(Q^{2}\right)$, and the deuteron radius, which is expected to hold in any case at very small $Q^{2}$, is found to extend to higher $Q^{2}$, up to $10 \mathrm{fm}^{-2}$. Knowing that there is for local models an empirical relation between the radius and the asymptotic normalization, $A_{S}$, the above observations raises the question of which model is the best with this respect, taking into account that this quantity is not changed in the transformations we performed.

The examination of the quadrupole form factor, especially at $Q^{2}=0$, where it identifies to the quadrupole moment, $Q_{D}$, also shows interesting features. For this one, contributions involving the non-locality can amount to $1 \%$, which is less than the difference between the upper value obtained with the Paris model $\left(0.279 \mathrm{fm}^{2}\right)$ and the lower ones (around $\left.0.270-0.271 \mathrm{fm}^{2}\right)$, obtained with the Nij1, Nij2, Reid93, Bonn-CD models. Curiously, there is no direct relation to the value of the ratio, $\eta=A_{D} / A_{S}$, which mainly determines this moment. After the effect of the non-locality is accounted for, which mainly affects values obtained with the Bonn models with respect to the other ones, we observe that the quadrupole moments are exactly in the same order as the $\eta$ values and not far to be proportional to them. Accounting for the non-locality effects therefore restores some regularity that was lost when considering predictions of Bonn models.

Throughout this paper, we ignored comparison with measurements, being essentially concerned by the comparison of theoretical predictions. We believe it is important in discriminating between models to separate effects that are due simply to a mathematical representation of the interaction, with more or less non-locality, and effects that have their origin in genuine aspects of the models. In making a comparison to measurements, one has first to determine among the various approximately phase-shift equivalent descriptions of the NN interaction which one is the more realistic, in the sense of relying on degrees of freedom that are as little effective as possible. Then, one has to also account for contributions from two-body currents corresponding to this optimal model. Some work along these lines has recently been done with the aim to extract the neutron charge form factor from the knowledge of the deuteron quadrupole form factor [23].

We are very grateful to R. Machleidt, C. Elster and R. Schiavella for providing us with material that has been employed here or has been useful in analyzing our own results.

\section{APPENDIX}


Induced contributions to the interaction, $\Delta V$

When the non-local terms of the form $\left\{\mathbf{p}^{2} / M, \tilde{W}\right\}$ are removed from an interaction model to transform it into a local potential, local terms of the same order in the interaction are generated. Their expression is given here for the scalar and tensor parts. For the first one, there is a factor 2 change with what we give in Ref. [13]. The new convention now agrees with the definition of the non-local term in Ref. [7]. For the second one, we give an expression which evidences its tensor character. Due to relations between $V_{0}$ and $W_{S}$, or $V_{1}$, $V_{2}$ and $W_{T}$ for the scalar and tensor non-local terms respectively (see Eqs. 13 , and 18, 19), other expressions could be obtained. Those given below are among the simplest we could find:

$$
\begin{aligned}
\Delta V_{S}^{0}= & -\frac{1}{2 M} W_{S}^{\prime \prime}+\frac{1}{M}\left\{L^{2}, \frac{V_{0}^{\prime}}{r}\right\} \\
\Delta V_{T}^{0}= & \frac{2}{M}\left\{S_{12}(\vec{L}), \frac{V_{2}}{r^{2}}\right\}+\frac{1}{M}\left\{L^{2}, S_{12}(\hat{r}) r\left(\frac{V_{1}}{r^{2}}\right)^{\prime}\right\} \\
& -\frac{1}{2 M} S_{12}(\hat{r})\left(W_{T}^{\prime \prime}+12 \frac{V_{2}}{r^{2}}\right),
\end{aligned}
$$

where

$$
S_{12}(\vec{A})=\frac{1}{2}\left(\vec{\sigma}_{1} \cdot \vec{A} \vec{\sigma}_{2} \cdot \vec{A}+\vec{\sigma}_{2} \cdot \vec{A} \vec{\sigma}_{1} \cdot \vec{A}\right)-\frac{1}{3} \vec{\sigma}_{1} \cdot \vec{\sigma}_{2} \vec{A}^{2}
$$

with $\vec{A}=\vec{L}$ or $\vec{A}=\hat{r}$.

As can be noticed, removing non-local terms introduces terms dependent on the total angular momentum, in agreement with the expectation that the phase-shift equivalent potential is local, wave by wave however. This is especially transparent for the scalar part. It is also noticed that the global range is not affected, contrary to the removing of off-shell effects, but the potential is somewhat more singular due to the presence of derivatives of $V_{0}, V_{1}, V_{2}$, or $W_{S}$ and $W_{T}$ in the above expressions. Particular caution may be therefore required in making qualitative statements concerning the effect of these terms. For instance, in case where $W_{S}$ would be given by a function, $\exp (-\mu r)$, the term involving the second derivative in Eq. (35) would produce a contribution that is attractive at large distances. However, it also produces a repulsive $\delta(r)$ term that will show up at very short distances. This one, often discarded, can give rise to the suppression of the S-state wave function that local models often evidence in this range. Although the singular character of the correction to the interaction does not necessarily prevent one to perform meaningful calculations, we felt better to cut-off the short-range part of the non-local interaction. In this way, we perhaps underestimate some of the effects but those 
we calculate are thus determined by the longer range part of the force, which is physically better known.

\section{Contributions to the static moments}

We here give the corrections to the static moments that can be obtained a direct calculation $\left(P_{D}\right)$ or from Eqs. (33) for the form factors in the limit $Q \rightarrow 0\left(\Delta<r^{2}>, \Delta Q\right)$.

$$
\begin{aligned}
& \Delta P_{D}=\frac{\sqrt{8}}{3} \int_{0}^{\infty} d r\left(\left(W_{T}(r)-6 V_{2}(r)\right) u(r) w(r)\right. \\
& \left.\left.+2 r\left(V_{1}(r)+2 V_{2}(r)\right) u^{\prime}(r) w(r)\right)\right), \\
& \Delta<r^{2}>_{S}=\frac{1}{2} \int_{0}^{\infty} d r r^{2} V_{0}(r)\left(u^{2}(r)+w^{2}(r)\right) \\
& \Delta<r^{2}>_{T}=\frac{\sqrt{8}}{3} \int_{0}^{\infty} d r r^{2}\left(V_{1}(r)+2 V_{2}(r)\right) \\
& \times\left(u(r) w(r)-\frac{1}{\sqrt{8}} w^{2}(r)\right), \\
& \Delta Q_{D}^{S}=\frac{\sqrt{2}}{5} \int_{0}^{\infty} d r r^{2} V_{0}(r)\left(u(r) w(r)-\frac{1}{\sqrt{8}} w^{2}(r)\right), \\
& \Delta Q_{D}^{T}=\frac{2}{5} \int_{0}^{\infty} d r r^{2}\left(V_{2}(r)\left(w^{2}(r)-u^{2}(r)+\frac{1}{\sqrt{2}} u(r) w(r)\right)\right. \\
& \left.-\frac{1}{3}\left(V_{1}(r)+2 V_{2}(r)\right)\left(u^{2}(r)+\frac{3}{2} w^{2}(r)-\sqrt{2} u(r) w(r)\right)\right) .
\end{aligned}
$$

\section{About another non-local term}

We here consider a non-local term of the form:

$$
V_{N L}=\left\{\frac{\overrightarrow{\sigma_{1}} \cdot \vec{p} \overrightarrow{\sigma_{2}} \cdot \vec{p}}{M}, W\right\}
$$

This one may arise from approximating the term given by Eq. (34) when 
treated in configuration space (order $1 / M^{4}$ ). It could also come from the exchange of a $a_{1}$ meson at the order $1 / M^{2}$. It contains both a scalar part, which has a form identical to the one discussed in the main text of the paper and a tensor part. This last term can be transformed into a local one at the lowest order, using the same transformation as for the other non-local tensor term (17). The functions $V_{1}$ and $V_{2}$ now fulfill the relation:

$$
\begin{aligned}
V_{1}^{\prime}(r)+V_{2}^{\prime}(r) & =0, \\
V_{1}(r)+V_{2}(r)+\left(r V_{2}(r)\right)^{\prime} & =W(r) .
\end{aligned}
$$

The above system of equations can be formally solved with the result:

$$
\begin{aligned}
& V_{2}(r)=-\frac{1}{r} \int_{r}^{\infty} \frac{W\left(r^{\prime}\right)}{r^{\prime}} d r^{\prime}, \\
& V_{1}(r)=-V_{2}(r) .
\end{aligned}
$$

\section{References}

[1] L.C. Alexa et al., Phys. Rev. Lett. 82 (1999) 1374.

[2] D. Abbott et al., Phys. Rev. Lett. 82 (1999) 1379.

[3] E. Brash, plenary talk given at BARYON2002, Newport News (March 4-8, 2002), to be published in the proceedings.

[4] M. Zuilhof and J.Tjon, Phys. Rev. C24 (1981) 736; P.L. Chung, F.Coester, B.D. Keister, W.N. Polyzou, Phys. Rev. C37 (1988) 2000; J.W. Van Orden, N. Devine and F. Gross, Phys. Rev. Lett. 75 (1995) 4369; D.R. Phillips, S.J. Wallace and N.K. Devine, Phys. Rev. C58 (1998) 2261; J. Carbonell and V.A. Karmanov, Eur. Phys. J. A 6 (1999) 9; F.M. Lev, E. Pace and G. Salmé, Phys. Rev. C62 (2000) 064004; T.W. Allen, W.H. Klink, W.N. Polyzou, Phys. Rev. C63 (2001) 034002.

[5] P. Doleschall and I. Borbély, Phys. Rev. C62 (2000) 054004.

[6] R. Machleidt, K. Holinde and Ch. Elster, Phys. Rep. 149 (1987) 1; R. Machleidt, Adv. in Nucl. Phys. 19 (1989) 189; R. Machleidt, Phys. Rev. C63 (2001) 024001.

[7] M. Lacombe et al., Phys. Rev. C21 (1980) 861.

[8] V.G.J. Stoks et al., Phys. Rev. C49 (1994) 2950.

[9] M. Lacombe et al., Phys. Rev. C65 (2002) 034004.

[10] J.L. Friar, Ann. of Phys. 104 (1977) 380. 
[11] J.L. Friar, Phys. Rev. C22 (1980) 796.

[12] B. Desplanques and A. Amghar, Z. Phys. 344 (1992) 19.

[13] A. Amghar and B. Desplanques, Nucl. Phys. A585 (1995) 657.

[14] H.V. von Geramb, Lectures notes in physics, Vol. 427 (1994); B.F. Gibson, H. Kohlhoff and H.V. von Geramb, Phys. Rev. C51 (1995) R465.

[15] J. Forest, Phys. Rev. C61 (2000) 034007.

[16] C. Elster et al., Few-Body Systems 21 (1996) 25.

[17] J.L. Friar, Phys. Rev. C60 (1999) 04002.

[18] V.G.J. Stoks, R. Timmermans and J.J. de Swart, Phys. Rev. C47 (1993) 512.

[19] T.E.O. Ericson, B. Loiseau and A.W. Thomas, hep-ph/0009312, and Nucl. Phys. A684 (2001) 380c.

[20] R.V. Reid, Ann. of Phys. 50 (1968) 411.

[21] R.B. Wiringa, V.G.J. Stoks and R. Schiavella, Phys. Rev. C51 (1995) 38.

[22] A. Amghar, B. Desplanques and L. Theussl, Nucl. Phys. A694 (2001) 439.

[23] R. Schiavella and I. Sick, Phys. Rev. C64 (2001) 041002(R). 